\title{
The Structure of a Turbulent Flow in a Channel of Complex Shape
}

GEOLOGICAL SURVEY PROFESSIONAL PAPER 983

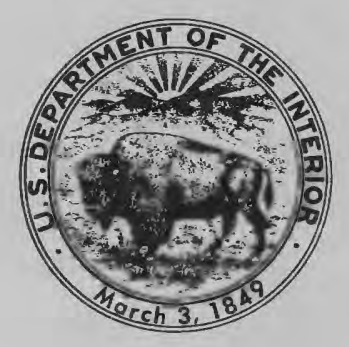





\section{The Structure of a Turbulent Flow in a Channel of Complex Shape}

By H. J. TRACY

GEOLOGICAL SURVEY PROFESIONAL PAPER 983

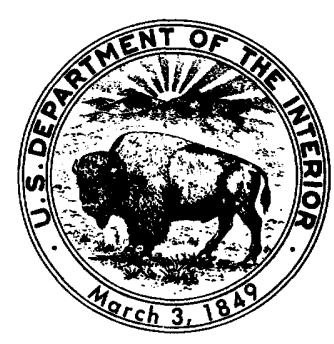

UN ITED STATES GOVERNMENT OFFICE, W ASHINGTON:1976 


\section{UNITED STATES DEPARTMENT OF THE INTERIOR}

THOMAS S. KLEPPE, Secretary

\section{GEOLOGICAL SURVEY}

\section{v. E. McKelvey, Director}

Library of Congress Cataloging in Publication Data

Tracy, Hubert Jerome, 1918-

The structure of a turbulent flow in a channel of complex shape.

(Geological Survey Professional Paper 983)

Bibliography: p. 24.

Supt. of Docs. no.: I $19.16: 983$

1. Turbidity. 2. Channels (Hydraulic engineering) I. Title. II. Series: United States Geological Survey Professional Paper 983. QE75.P9 no. 983 [GB665]

For sale by the Superintendent of Documents, U.S. Government Printing Office

Washington, D.C. 20402

Stock Number 024-001-02875-1 


\section{CONTENTS}

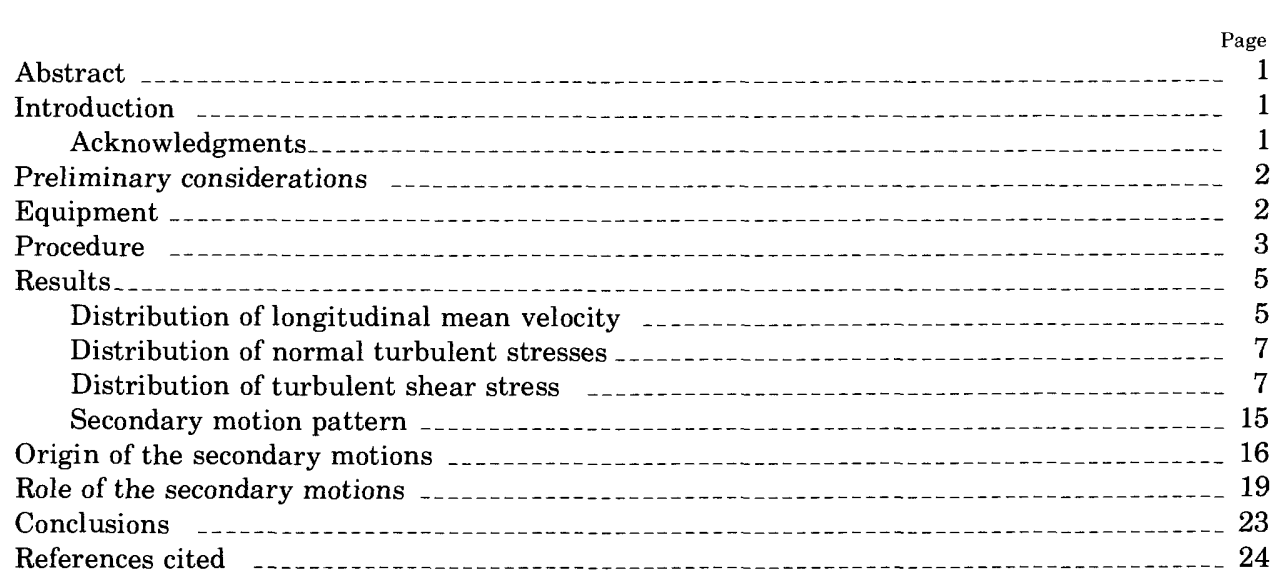

\section{ILLUSTRATIONS}

1. Schematic drawing showing components of turbulent stress on an elementary particle

2. Schematic drawing of test conduit

3. Schematic drawing of channel section

4. Graph showing mean pressure along channel

5. Graph showing distribution of longitudinal velocity near wall

6. Graph showing distribution of longitudinal velocity away from wall

7.-12. Graphs showing distributions of turbulence quantities:

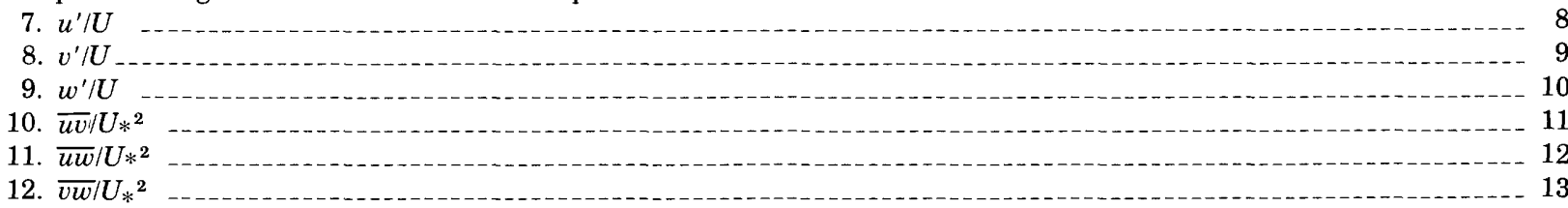

12. $\overline{v w} / U_{*}{ }^{2}$

14. Graph showing distributions of correlation coefficients $\overline{u v} / u^{\prime} v^{\prime}$

15. Graph showing distributions of correlation coefficients $\overline{u w} / u^{\prime} w^{\prime}$

16. Chart showing secondary motions in channel

17. Graph showing distribution of $\overline{v^{2}}-\overline{w^{2}}$

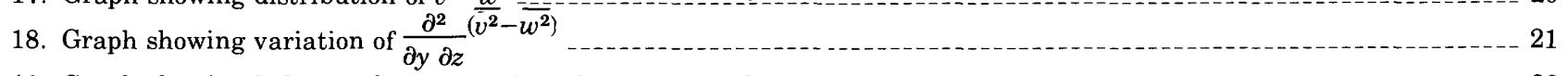

19. Graph showing balance of first equation of motion at $y / d=0.08$

20. Graph showing distribution of sum of turbulent shear farces

\section{SYMBOLS}

$C=$ constant used to describe velocity variation

$d=$ half height of channel, 6 in. $(15.2 \mathrm{~cm})$

$D=$ height of channel

$p=$ piezometric pressure; $P_{m}$ is pressure of measuring station

$R=$ hydraulic radius, ratio of cross-sectional area to section perimeter

$t=$ time

$U, V, W=$ mean velocity parallel to $x, y, z$ directions, respectively

$U_{o}=$ maximum value of longitudinal mean velocity in the channel

$U_{*}=$ "friction" velocity, $\sqrt{\tau_{o} / \rho}$

$u, v, w=$ instantaneous values of velocity fluctuations parallel to the

$x, y, z$ directions, respectively $u^{\prime}, v^{\prime}, w^{\prime}=$ root mean square of velocity fluctuations $u, v, w$, respectively

$x=$ longitudinal coordinate along channel, $x=0$ correspondents to test section

$y=$ vertical coordinate, $y=0$ corresponds to channel fioor $z=$ lateral coordinate, $\mathrm{z}=0$ corresponds to channel sidewall $\xi=$ rotation in $y-z$ plane

$\mu=$ dynamic viscosity

$\rho=$ density

$\tau_{0}=$ average shear stress on boundary

$v=$ kinematic viscosity, $\mu / \rho$ 



\title{
THE STRUCTURE OF A TURBULENT FLOW IN A CHANNEL OF COMPLEX SHAPE
}

\author{
By H. J. Tracy
}

\section{ABSTRACT}

Measurements of the Reynolds stresses and the mean motion pattern were made in a uniform turbulent motion in a conduit consisting of a large, nearly square section joined by a smaller rectangular section. The results indicate that the boundary shearing stress is nearly constant over large segments of the boundaries. It is shown that the magnitudes of the lateral and the vertical components of turbulence are not the same near a boundary and that the component normal to the boundary is smaller than the component parallel to the boundary. The difference in the two components in the corner regions of the channel produces secondary mean motions in the plane of the channel section. The strength of the motion depends upon the angle subtended by the corner.

The magnitude of a component of turbulent shearing stress is a function of boundary proximity. In the corner regions, the effect of the combination of the two walls is to create large shear stress gradients, or forces. The forces are frequently larger than those of the pressure. Depending upon the variation of the mean streamwise velocity, the shear force may either oppose or act in the same direction as the pressure force.

A principal function of the secondary motions is to transfer momentum into the corner regions and, elsewhere, to compensate for the excess force due to the shear gradients. In the absence of the secondary motions, the fluid must stagnate and separate from the boundaries in certain regions and be greatly accelerated in others.

The secondary motions are conventionally described in terms of symmetrical rotations in cells bounded by the corner bisectors. The measured motion pattern is at variance with this view, unless the symmetry is confined to a very local region.

\section{INTRODUCTION}

The mean flow characteristics are usually the ultimate concern of those who deal with the movement of fluids in open channels and in closed conduits. Often, engineering solutions of satisfactory accuracy may be obtained from equations of the Manning or Chezy type, or from one-dimensional energy and momentum considerations. As the body of experimental information grows, however, it becomes increasingly evident that the rational interpretation of many of the phenomena that are encountered depends upon a better knowledge of the internal structure of the flow than is presently available. In particular, much of the current work in this field is devoted to an understanding of the role of the turbulent fluctuations in shaping the mean flow field.

Presently, comprehensive data are limited to simple examples of turbulent flow. These include (1) a twodimensional flow (Laufer, 1951), (2) an axially symmetrical flow (Laufer, 1954), and flow in (3) a square conduit (Brundrett and Baines, 1964), (4) a rectangular conduit (Tracy, 1965), and in (5) a trapezoidal conduit (Rodet, 1960); all examples are for a uniform flow condition. The present study was carried out in a channel having a boundary form more complex in configuration than any of those listed above. Its principal added feature is a corner subtending an angle greater than 180 degrees.

In section, the test channel consisted of a larger rectangular channel joined by a smaller rectangular overbank channel. The selection of this arrangement was influenced by the procedures commonly used in natural stream channel flow computations. The section of natural stream channels is frequently subdivided, and each subchannel is subsequently treated as an independent entity for flow computation purposes. The subdivisions are usually made at major changes in boundary alignment and at points on the boundary at which the boundary roughness changes significantly. The test data, though restricted in application by the somewhat idealized shape and uniformity of the laboratory channel, and by its smallness, should nevertheless yield information relevant to the validity of the subdivision procedure.

The objective of the investigation was a study of the turbulent and the secondary mean motions generated in the larger of the two channels, and an evaluation of the effect of the various corners of the section in terms of the momentum transport engendered by the motions. To this purpose, measurements were made of the mean motions, both primary and secondary, and of the relevant statistical quantities of the turbulence over the section. These, when substituted into the equations of motion, can be expected to show the manner in which a given flow adjusts to the configuration of its boundaries, even though many of the mechanisms by which this is accomplished are yet unknown.

\section{ACKNOWLEDGMENTS}

The study was carried out in a test facility located in 
the hydraulics laboratory of the Georgia Institute of Technology, under a cooperative agreement with the Geological Survey, U.S. Department of the Interior. D. W. Hicks assisted in the collection of the test data.

\section{PRELIMINARY CONSIDERATIONS}

The continuity equation and the Reynolds equation in rectangular coordinates for incompressible flow may be written as follows:

$$
\begin{gathered}
\frac{\partial U}{\partial x}+\frac{\partial V}{\partial y}+\frac{\partial W}{\partial z}=0 \\
\rho \frac{D U}{D t}=-\frac{\partial p}{\partial x}+\mu \nabla^{2} U-\rho\left(\frac{\partial \overline{u^{2}}}{\partial x}+\frac{\partial \overline{u v}}{\partial y}+\frac{\partial \overline{u w}}{\partial z}\right) \\
\rho \frac{D V}{D t}=-\frac{\partial p}{\partial y}+\mu \nabla^{2} V-\rho\left(\frac{\partial \overline{u v}}{\partial x}+\frac{\partial \overline{v^{2}}}{\partial y}+\frac{\partial \overline{v w}}{\partial z}\right) \\
\rho \frac{D W}{D t}=-\frac{\partial p}{\partial z}+\mu \nabla^{2} W-\rho\left(\frac{\partial \overline{u w}}{\partial x}+\frac{\partial \overline{v w}}{\partial y}+\frac{\partial \overline{w^{2}}}{\partial z}\right)
\end{gathered}
$$

where the superscript bar is used to denote a time average; $D / D t$ refers to the total derivative

$$
\frac{D}{D t}=\frac{\partial}{\partial t}+U \frac{\partial}{\partial x}+V \frac{\partial}{\partial y}+W \frac{\partial}{\partial z},
$$

and $\nabla^{2}$ to the operator

$$
\nabla^{2}=\frac{\partial^{2}}{\partial x^{2}}+\frac{\partial^{2}}{\partial y^{2}}+\frac{\partial^{2}}{\partial z^{2}}
$$

$U, V$, and $W$ are the components of mean velocity in the $x, y$, and $z$ directions, respectively, and $u, v$, and $w$ are the correspondingly directed values of the turbulent velocity fluctuations. The mean pressure at a point is $p$, and $\rho$ and $\mu$ are the density and dynamic viscosity of the fluid. The normal stresses $\rho \overline{u^{2}}, \rho \overline{v^{2}}$, and $\rho \overline{w^{2}}$ and the tangential stresses $\rho \overline{u v}, \rho \overline{u w}$, and $\rho \overline{v w}$ are orientated as shown in figure 1.

In a steady, uniform motion, the velocity field is independent of the longitudinal coordinate $(x)$ and of time. The equations therefore become:

$$
\begin{gathered}
\frac{\partial V}{\partial y}+\frac{\partial W}{\partial z}=0 \\
\rho\left(V \frac{\partial U}{\partial y}+W \frac{\partial U}{\partial z}\right)=-\frac{\partial p}{\partial x}+\mu\left(\frac{\partial^{2}}{\partial y^{2}}+\frac{\partial^{2}}{\partial z^{2}}\right) U-\rho\left(\frac{\partial \overline{u v}}{\partial y}+\frac{\partial \overline{u w}}{\partial z}\right) \\
\rho\left(V \frac{\partial V}{\partial y}+W \frac{\partial V}{\partial z}\right)=-\frac{\partial p}{\partial y}+\mu\left(\frac{\partial^{2}}{\partial y^{2}}+\frac{\partial^{2}}{\partial z^{2}}\right) V-\rho\left(\frac{\partial \overline{v^{2}}}{\partial y}+\frac{\partial \overline{v w}}{\partial z}\right) \\
\rho\left(V \frac{\partial W}{\partial y}+W \frac{\partial W}{\partial z}\right)=-\frac{\partial p}{\partial z}+\mu\left(\frac{\partial^{2}}{\partial y^{2}}+\frac{\partial^{2}}{\partial z^{2}}\right) W-\rho\left(\frac{\partial \overline{v w}}{\partial y}+\frac{\partial \overline{w^{2}}}{\partial z^{2}}\right)
\end{gathered}
$$

The abbreviated equations describe the motion to be considered here and, except possibly for the terms involving the viscosity, may not be further simplified. The viscous terms are of relative significance in a fully
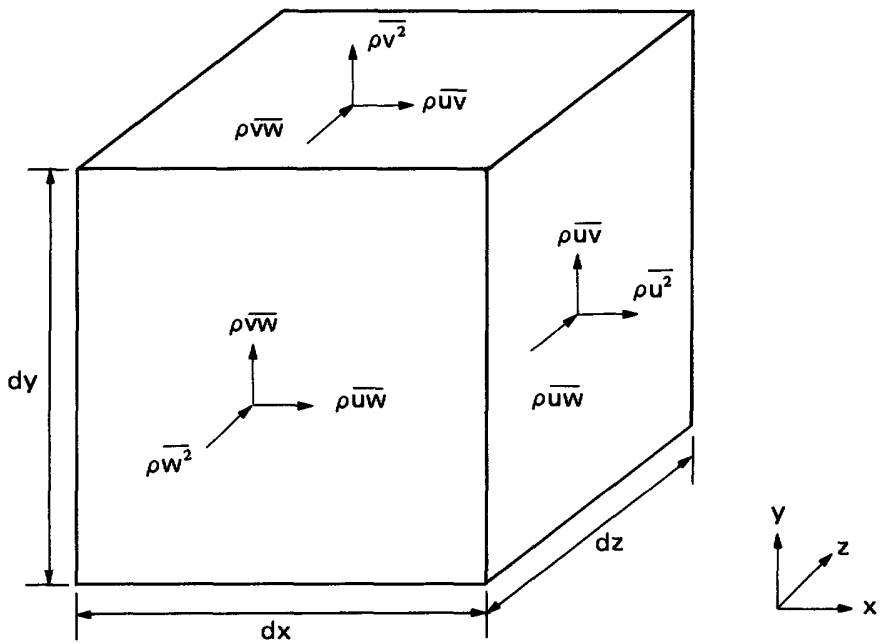

FiguRE 1.-Components of turbulent stress on an elementary particle.

developed turbulent flow only in regions very close to a boundary.

\section{EQUIPMENT}

Test Conduit.-The experimental measurements were made in the closed-circuit air tunnel shown schematically in figure 2. A cross section of the test channel is shown in figure 3 .

The test channel was constructed of commercial aluminum plate and standard aluminum structural shapes. The transition section at the entrance to the channel and at its end were formed from aluminum sheet. The return section and the upstream and downstream turn boxes were constructed of interior grade plywood. The measurement cross section was located $84 \mathrm{ft}(25.6 \mathrm{~m})$ downstream from the end of the upstream transition section.

Air was supplied to the tunnel from a centrifugal blower powered by a 15 horsepower $(11.2 \mathrm{~kW})$ variable-speed direct-current motor. Blower speed, over a continuous range, was controlled by variations in the current supplied to the motor. The variable speed feature of the motor and blower was utilized for inplace calibration of the hot-wire probes used during the investigation.

Hot-wire equipment. -A commercially "available two-channel DISA Electronics' ${ }^{1}$ constant-temperature hot-wire instrument was used during the study. Each channel consisted of a $55 \mathrm{D} 01$ anemometer unit, a $55 \mathrm{D}$ 10 linearizer, and a 55 D 25 auxiliary unit. Outputs were measured with a $55 \mathrm{D} 30 \mathrm{DC}$ meter and a $55 \mathrm{D} 35$ RMS meter. A model 823 Fluke voltmeter, in conjunction with a wave generator, was used as a voltage

${ }^{1}$ The use of brand names in this report is for identification purposes only and does not imply endorsement by the U.S. Geological Survey. 


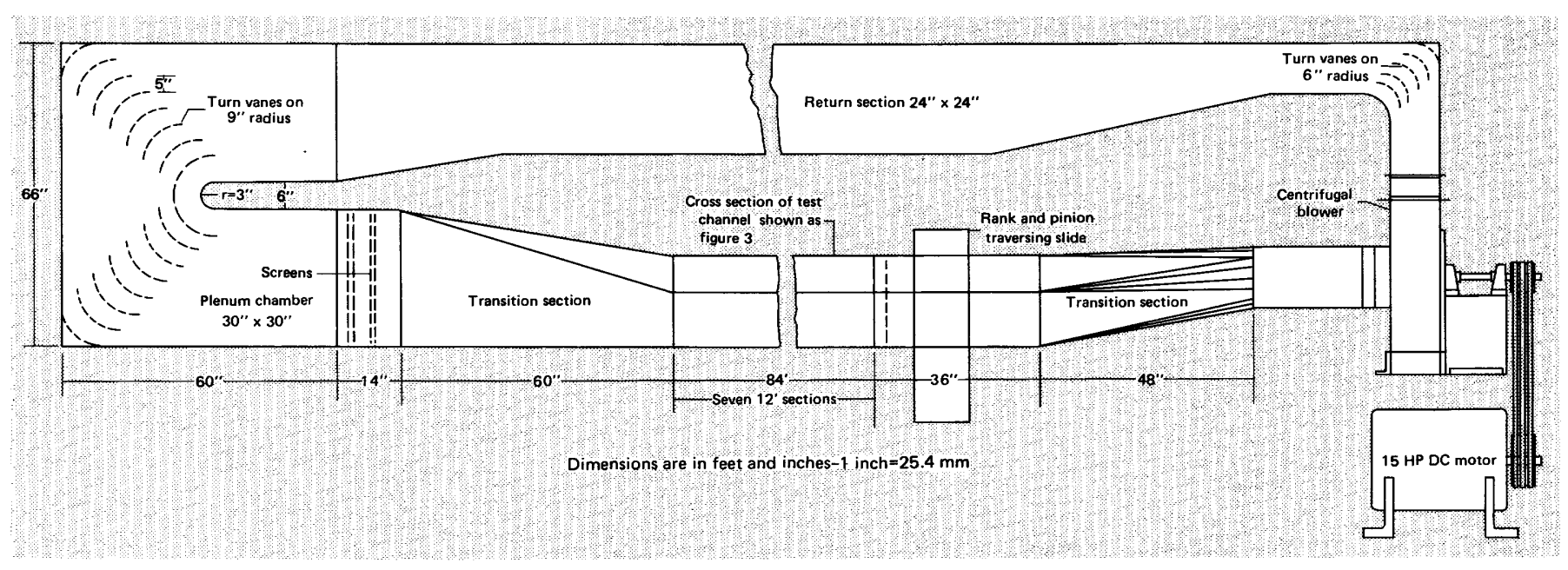

FIGURE 2.-Schematic of test conduit.

standard for the calibration of both the $\mathrm{AC}$ and the DC DISA meters.

The hot wires were either $0.0001 \mathrm{in} .(0.0025 \mathrm{~mm})$ or 0.0002 in. $(0.0051 \mathrm{~mm})$ diameter platinum wire and were used either singly or in $x$ arrays depending upon the variable to be measured. Wire length for the single-wire probes was $0.030 \mathrm{in} .(0.76 \mathrm{~mm})$, and for the $x$ arrays, 0.042 in. $(1.06 \mathrm{~mm})$. The $x$ array wires subtended a central angle of 90 degrees and were separated by a distance of about $0.020 \mathrm{in} .(0.51 \mathrm{~mm})$. The wires, in both cases, were supported by fine sewing needles projecting from one end of short lengths of small diameter brass tubing.

Traversing device.-The probes were traversed over the channel section by independent vertical and horizontal mechanisms. Vertical movement was registered on scales attached to rack-and-pinion mounted plates inserted into the two vertical faces of a transparent working section on one side of the channel at the measurement station. Horizontal movement was measured on a sliding scale extending through and perpendicular to the plates. The probe support was fastened to the end of the sliding scale.

Zero readings of the traverse scales were found by placing the probe tip arbitrarily close to the vertical and horizontal walls and by then measuring the distance between the tip and its image in the appropriate wall on an ocular micrometer. The traversing scale reading corresponding to one-half of the observed distance marked the location of the wall.

\section{PROCEDURE}

The test channel was constructed so that its axis of symmetry was vertical, with the smaller channel above the larger. Cartesian coordinates are used for point location and are orientated with $x$ along the length of the channel, $y$ in the vertical direction, and $z$ horizontally directed. For economy in the required number of figures and for ease of comparison, the measurements in the upper and in the lower halves of the larger channel are often shown together. For this purpose only, the origin of coordinates for $y$ and $z$ for all data in the upper half of the channel is located at the upper left corner of the channel. For data in the lower half, the origin for $y$ and $z$ is the lower right corner. For any other purpose, only the lower origin is used, and $y$ increases continuously from the lower to the upper boundary. For $x$, the origin is at the measuring station and is positive in the downstream direction. The root-mean-square values of the turbulent components in the $x, y$, and $z$ directions are denoted by $u^{\prime}, v^{\prime}$, and $w^{\prime}$. The height of the lower channel is $D$. The halfheight, $d$, is used as a reference length to nondimensionalize the location of the points at which data were taken.

The channel was sufficiently long $(L / D$ greater than 80) for full development of the flow at its downstream end. Velocity measurements were made on each side of the axis of symmetry to verify that the motion was symmetrical with respect to the axis. All tests were made with the maximum channel velocity maintained constant at $75 \mathrm{ft} / \mathrm{s}(22.9 \mathrm{~m} / \mathrm{s})$, corresponding to a Reynolds number of about 440,000 , using $D$ as the appropriate length.

The longitudinal mean pressure was measured from piezometer taps located every $3 \mathrm{ft}(0.91 \mathrm{~m})$ along the channel for the last $45 \mathrm{ft}(13.7 \mathrm{~m})$ of length. The results are shown in figure 4 . The pressures are referenced to the pressure at the measuring station, $p m$.

Mean velocities were measured by small diameter total head tubes used in conjunction with wall piezometers and by hot wires. The total head tubes 


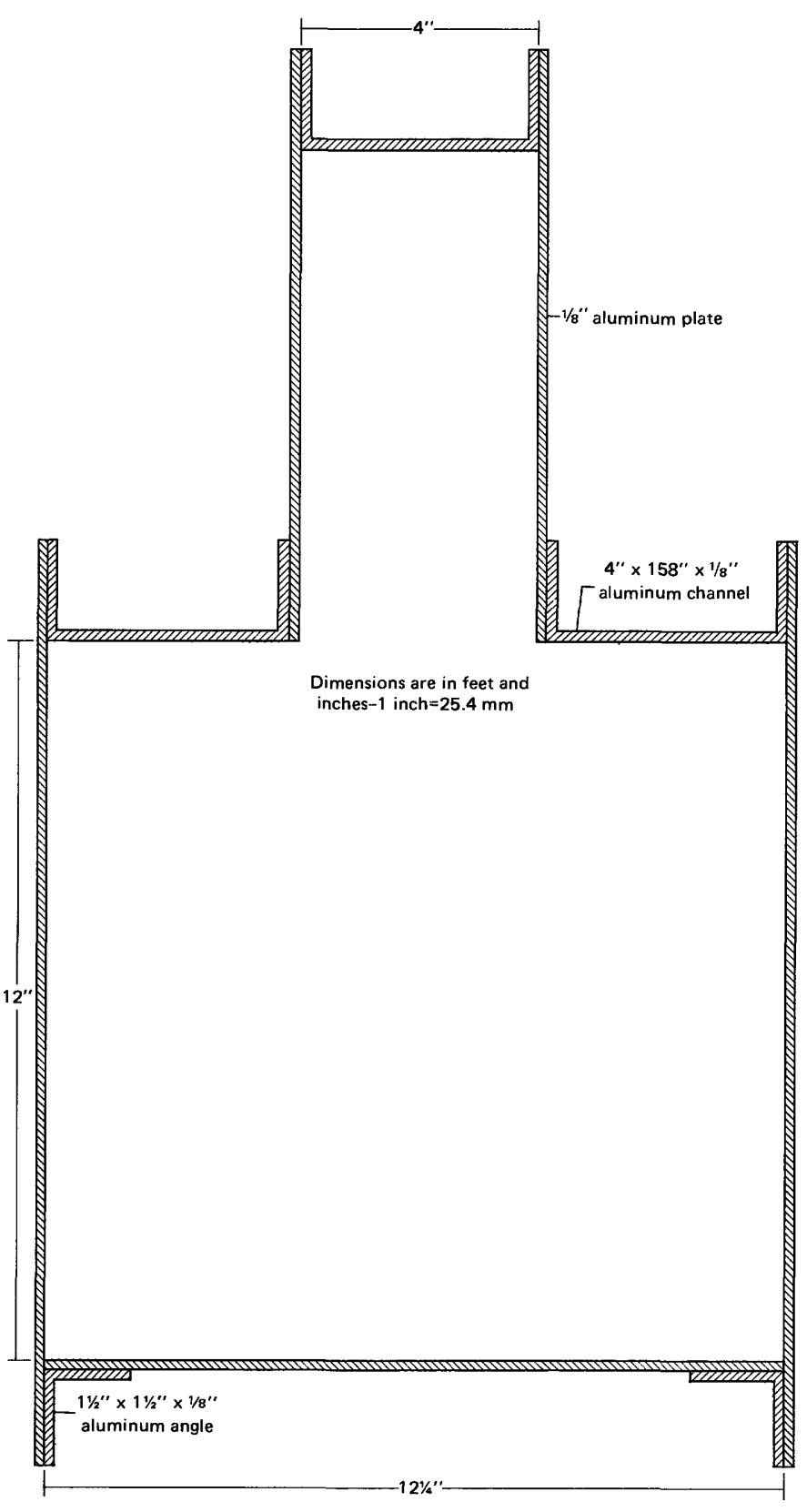

Figure 3.-Channel section.

were either $0.035 \mathrm{in} .(0.89 \mathrm{~mm})$ or $0.020 \mathrm{in} .(0.51 \mathrm{~mm})$ in diameter. The smaller diameter tubes were used for measurements in the vicinity of the wall.

The longitudinal mean velocity measurements were corrected for the effect of the turbulent velocity fluctuations by the relation

$U($ corrected $)=U($ measured $) \sqrt{1-\frac{\overline{u^{2}}+\overline{v^{2}}+\overline{w^{2}}}{U^{2}(\text { measured })}}$.

Hot-wire measurements, except those for $V$ and $W$, were obtained by standard techniques described by Schubauer and Klebanoff (1946), Hinze (1954), and

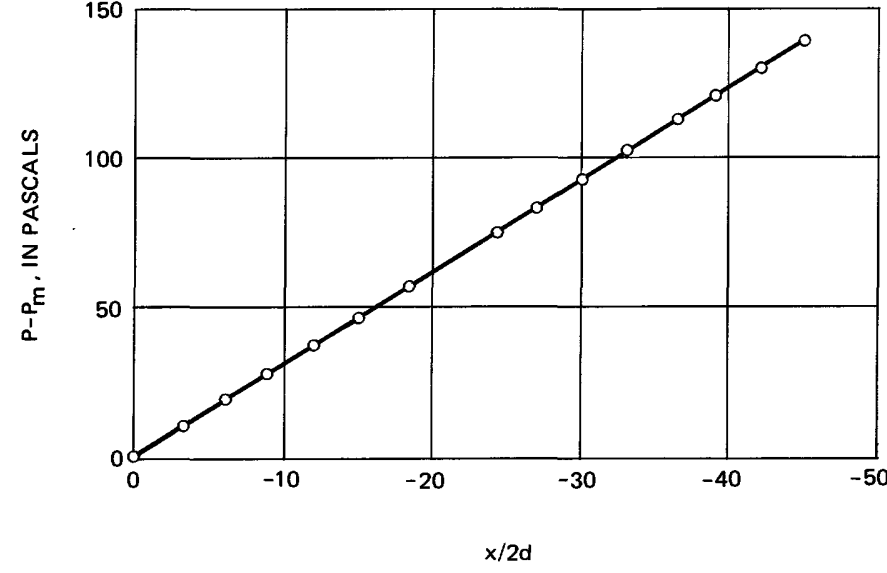

FIGURE 4.-Mean pressure along channel.

Rodet (1960). The measurement of $V$ and $W$ follows from the geometric relationships between $U, V$, and $W$ and their relative magnitudes and from certain characteristics of the hot wire. These are outlined below.

The secondary velocities are at right angles to the velocity, $U$, and are small relative to $U$. Their effect is a change in the local direction of the mean flow from the direction parallel to $U$. The angular deviation, in radian measure, from the $U$ direction is $V / U$ in the $x-y$ plane and $\mathrm{W} / \mathrm{U}$ in the $x-z$ plane.

The heat loss from a hot wire is a function of the magnitude of the component of velocity normal to the wire. The heat loss from each of two wires which are arranged in an $x$ configuration and which are independently maintained at a constant temperature thus depends upon the degree to which each wire is normal to the flow, or upon the angle which each subtends to the flow. Since the position of each wire relative to the other is fixed, a rotation of the array to increase the exposure of one of the wires will decrease the exposure of the other and thus change the heat-loss characteristics of each wire. Correspondingly, a change in the direction of the flow with the array in an unchanged angular position will have the same effect. Hence, a given array may be calibrated to sense a change in the angular direction of the flow by a procedure in which the heat loss difference between the two wires is determined as a function of flow angle and velocity. After calibration in this manner, the probe may be inserted into the flow and traversed throughout the section, maintaining the array in an invariant angular orientation. The difference between the losses can be related to $V / U$ for the array placed in the $x-y$ plane and to $W / U$ for the array in the $x-z$ plane by the results of the calibration.

On the other hand, the directional sensitivity of the $x$ array probe type was a source of considerable incon- 
venience during the measurement of the turbulent shearing stresses $\rho \overline{u v}, \rho \overline{u w}$, and $\rho \overline{v w}$. The measurements require that each wire of the array subtend the same angle with respect to the mean flow direction at each measurement point, which necessitated a pointto-point alinement of the angular position of the array to correspond to the variations in mean flow direction due to the superposed secondary motions. Equal voltages through each of the two matched wires indicated the proper setting to place each wire at the same angle to the mean flow. The response of the arrays to the normal stresses $\rho \bar{v}^{2}$ and $\rho \bar{w}^{2}$ is less critically dependent upon probe orientation.

Hot wires placed close to a boundary are affected by heat transfer into the boundary. The transfer is variable, depending upon boundary distance. Some compensation for the effect is provided by the constanttemperature feature of the mode of operation, which allows the transfer, and therefore the slope of the voltage-velocity relationships upon which the turbulence computations depend, to remain somewhat constant at a given wall distance, irrespective of velocity variation. Also, the large mean velocity gradients near a boundary are also a source of error. When parallel to the boundary, the wires of a two-wire array are exposed to velocity differences which may be quite large for wires separated by even small distances. When normal to a wall, the mean velocity distribution along the wires of single and two-wire arrays alike is nonuniform.

Adjustments have not been made to the hot-wire data to account for the boundary effects. The data closer to the wall than about $3 \mathrm{~mm}$ must thus be considered less reliable than elsewhere.

\section{RESULTS}

\section{DISTRIBUTION OF LONGITUDINAL MEAN VELOCITY}

The variation of the mean longitudinal component of velocity over the left one-half of the nearly rectangular portion of the test channel is shown in figures 5 and 6 . Each curve represents a traverse horizontally outward from the vertical wall. Velocity measurements close to the wall are shown in figure 5 and the remainder of the traverse to the channel centerline is shown in figure 6 . The vertical location $(y / d)$ of each traverse with respect to distance from the upper horizontal boundary or from the lower horizontal boundary is shown in the legend. Velocities in the lower quadrant of the channel are identified by open symbols. A slash mark through the symbol designates an observation in the upper quadrant. The half-channel height, $d$, is 6 in. $(152.4 \mathrm{~mm})$.

The distributions shown in figure 5 in the two parts of the channel superpose on the basis of $y / d$, except

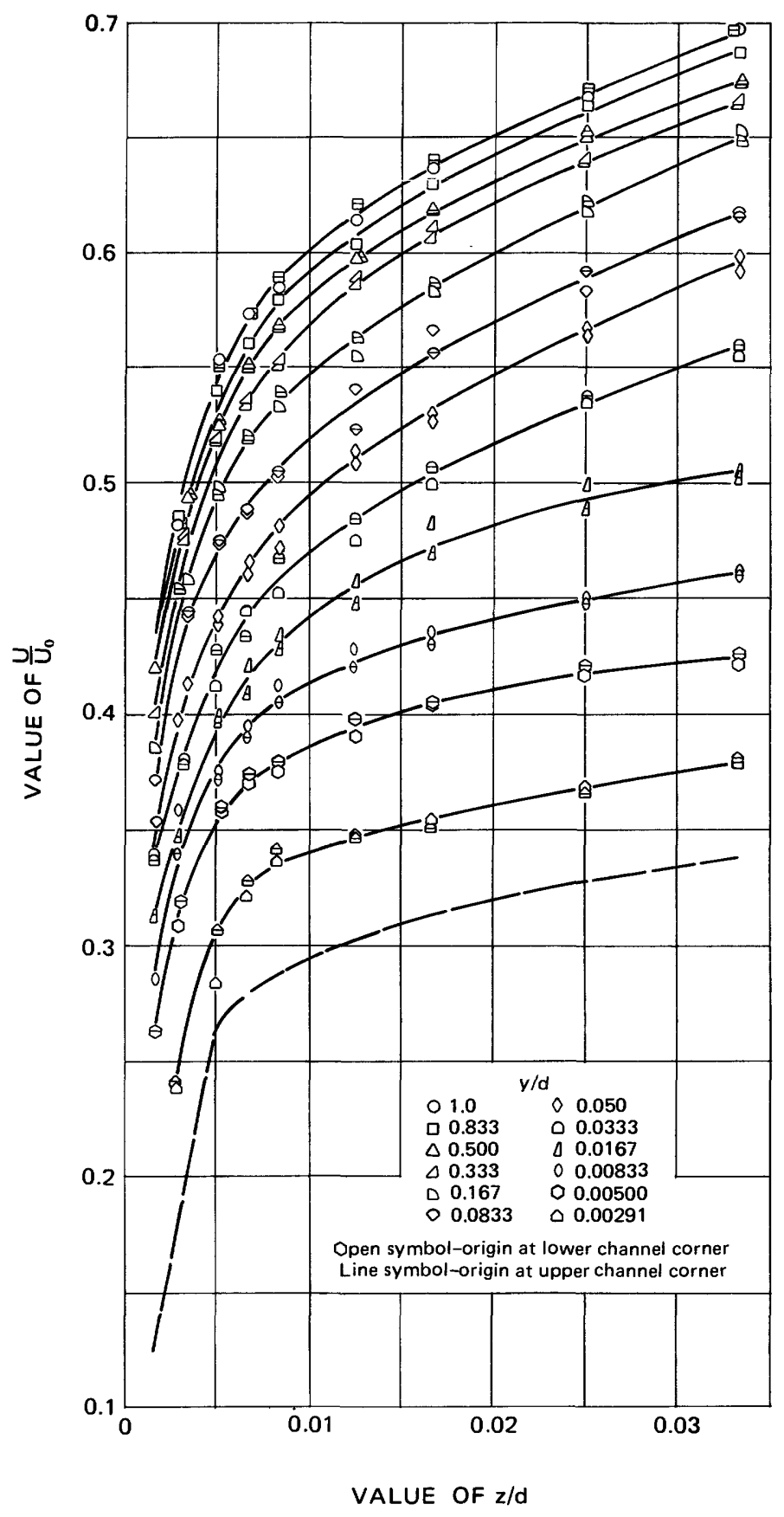

FIGURE 5.-Distribution of mean velocity near wall.

near $y / d=1.0$. The variation, to a good approxımation, can be represented by the equation

$$
\frac{U}{U_{o}}=C\left(\frac{y}{d}\right)^{\frac{1}{n}},
$$

with $n$ equal to about 7.5. The curves in the two quadrants of the channel diverge farther from the wall (fig. 6). The relatively greater number of observations near the horizontal boundaries tend to emphasize the differences in the flow in the two quadrants, whereas the differences are actually minimal outside a limited 


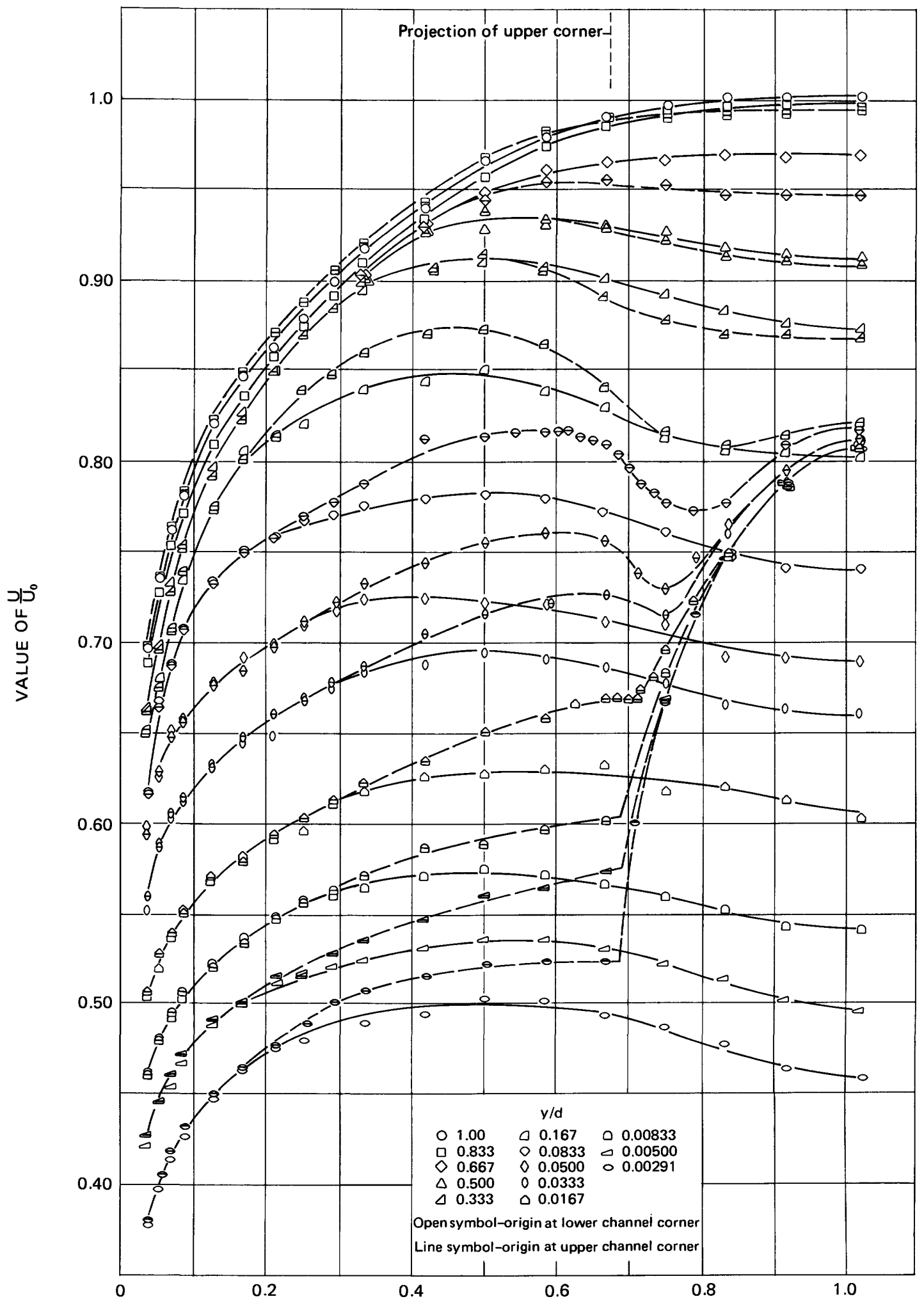

VALUE OF $z / d$

Figure 6.-Distribution of mean velocity away from wall. 
region $(y / d<0.17)$. The effect of the upper adjoining channel on the mean flow is essentially restricted to the vicinity of the junction between the two channels. The differences outside the limited region are primarily related to differences in the secondary motion patterns of the two quadrants.

The secondary motions are a system of mean circulations in the plane of the channel section, which transfer mass from one region to another region of the flow. If the velocities in the two regions are not the same, momentum is either gained or lost by the migrating mass, and a corresponding retarding or accelerating force is exerted on the flow during the course of the movement.

The decrease in velocity at the outer ends of many of the curves of figure 6 is an example of the effect of the circulations. The decrease can be explained by an upward motion (and the measurements of the motion to be shown later indicate this to be true) near $z / d=1.0$ near the lower horizontal boundary and by a downward motion in a corresponding location in the upper half of the channel. In each case, the motion is from a region of a lower velocity to one of a higher velocity, which retards the flow along its path. The motions, and their effects, are discussed in a later section of the report.

Although the larger channel is nearly square in shape, the coordinates $y$ and $z$ are not interchangable, even in the lower quadrant of the channel which is the most removed from the effects of the smaller channel. The characteristic decrease in velocity at the outer ends of many of the $U / U_{o}$ versus $z / d$ curves is not typical of the variation of $U / U_{o}$ versus $y / d$. The velocity increases in a continuous manner in a direction vertically outward from each of the horizontal boundaries to the effective horizontal centerline of the channel.

The channel section is not suitably proportioned to establish a region of two-dimensional flow. Nevertheless, except for some flattening at the outer end where $\partial U / \partial z$ is zero, the velocity curve for $y / d=1.0$ approximates a logarithmic distribution of the form

$$
\frac{U}{U_{*}}=C_{1}+C_{2} \log \frac{y U_{*}}{\nu},
$$

where $U_{*}$ is the "friction" velocity, $\sqrt{\tau o / \rho}$, and $v$ is the kinematic viscosity. The average shear stress at the channel boundaries, $\tau_{o}$, was computed from the measured longitudinal pressure gradient, $\frac{\partial p}{\partial x}$, and from the geometric properties of the flow section as follows:

$$
\tau_{o}=-\frac{\partial p}{\partial x} \mathbf{R}
$$

where $R$ is the ratio of cross-sectional area to section perimeter. The constants $C_{1}$ and $C_{2}$ are 4.5 and 6.6, respectively, which agree only approximately with those obtained by others in a two-dimensional flow. The differences are functions of channel aspect ratio (ratio of width to height) and boundary roughness.

\section{DISTRIBUTION OF NORMAL TURBULENT STRESSES}

The distributions of the measured values of $u^{\prime} / U$, $v^{\prime} / U$, and $w^{\prime} / U$ over the test channel are shown in figures 7, 8, and 9. As for the velocity measurements, the traverses at a common distance from the upper and from the lower horizontal boundaries are designated by the same symbol. The traverses in the upper half of the channel are distinguished from those in the lower half by a slash mark through the symbol. The $u^{\prime} / U$ values were computed from the response of a linearized anemometer, and the values of $v^{\prime} / U$ and $w^{\prime} / U$ from a non-linear signal.

The measurements represent the standard deviations of the velocity fluctuations in the three directions divided, in each case, by the mean longitudinal point velocity. The ratio is defined as the relative intensity of turbulence. The standard deviation is a measure of the spread or dispersion of the component about its mean-the greater the value, the larger the dispersion. Although they are a convenient and useful device, the measurements do not, obviously, completely describe the eddy formations.

The measured distributions show that the turbulence is nearly isotropic in the center of the channel and has its smallest values there. All three components become larger in any direction toward a boundary. The longitudinal component increases at a faster rate than the others and at the boundary attains roughly twice the magnitude of the larger of the remaining two.

A comparison of figures 8 and 9 indicates that $v^{\prime} / U$ increases at a faster rate than does $w^{\prime} / U$ near a vertical boundary and that the reverse is true near a horizontal boundary. A boundary acts, apparently, to restrain the eddy in the direction normal to the boundary, whereas the eddy is not restrained in the direction parallel to the boundary.

The results of Laufer $(1951,1954)$, Brundrett and Baines, (1964), and Tracy (1965) confirm these generalizations to a good approximation over a Reynolds number range of about 12,000 to nearly 500,000 .

\section{DISTRIBUTION OF TURBULENT SHEAR STRESS}

The turbulent cross-products $\overline{\mathrm{uv}}, \overline{\mathrm{uw}}$, and $\overline{\mathrm{vw}}$, after division by $\mathrm{U}_{*}{ }^{2}$, are shown in figures 10,11 , and 12 , respectively. They are referenced to coordinate origins 
STRUCTURE OF A TURBULENT FLOW IN A CHANNEL OF COMPLEX SHAPE

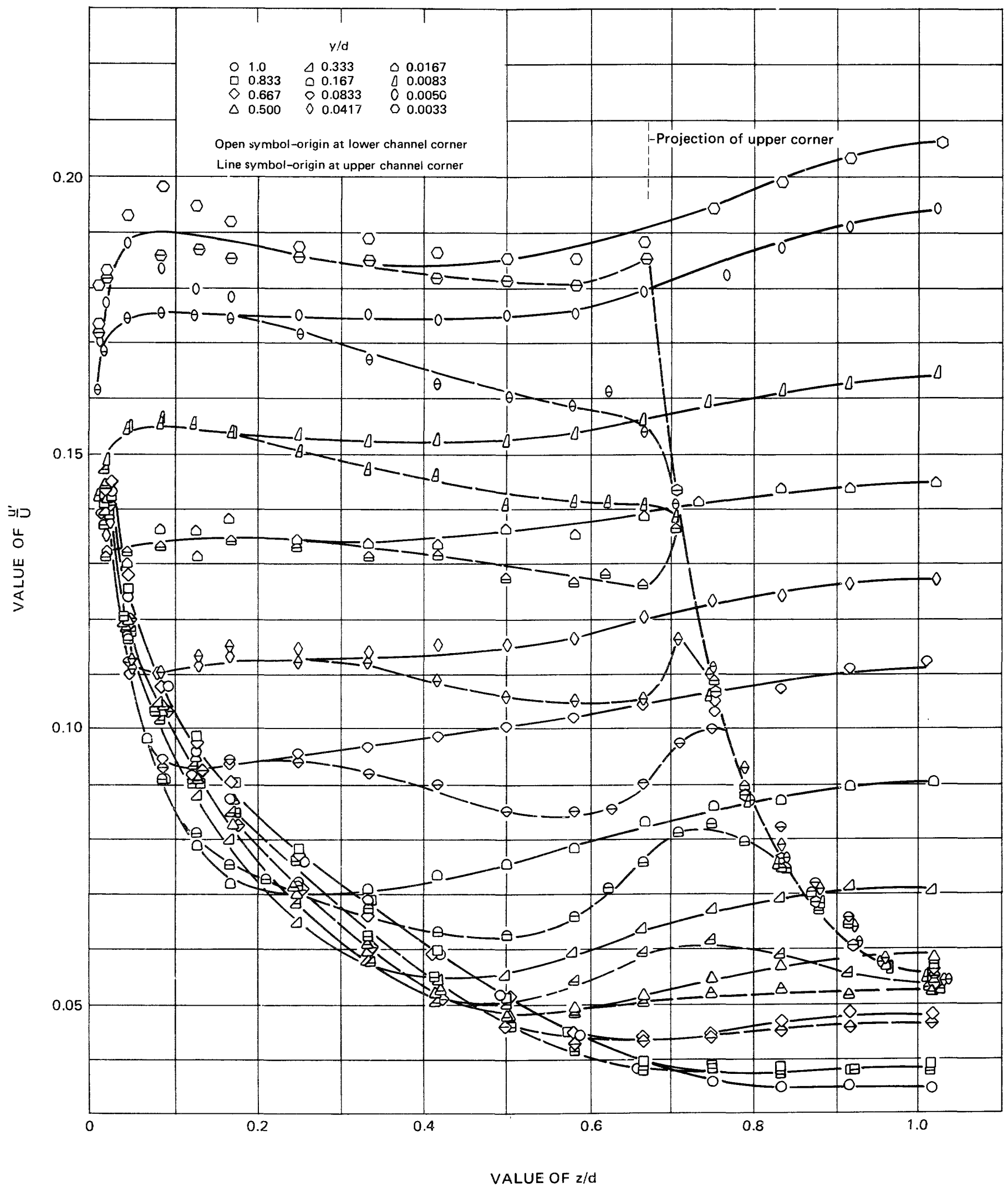

FigURE 7.-Distribution of $u^{\prime} / U$. 


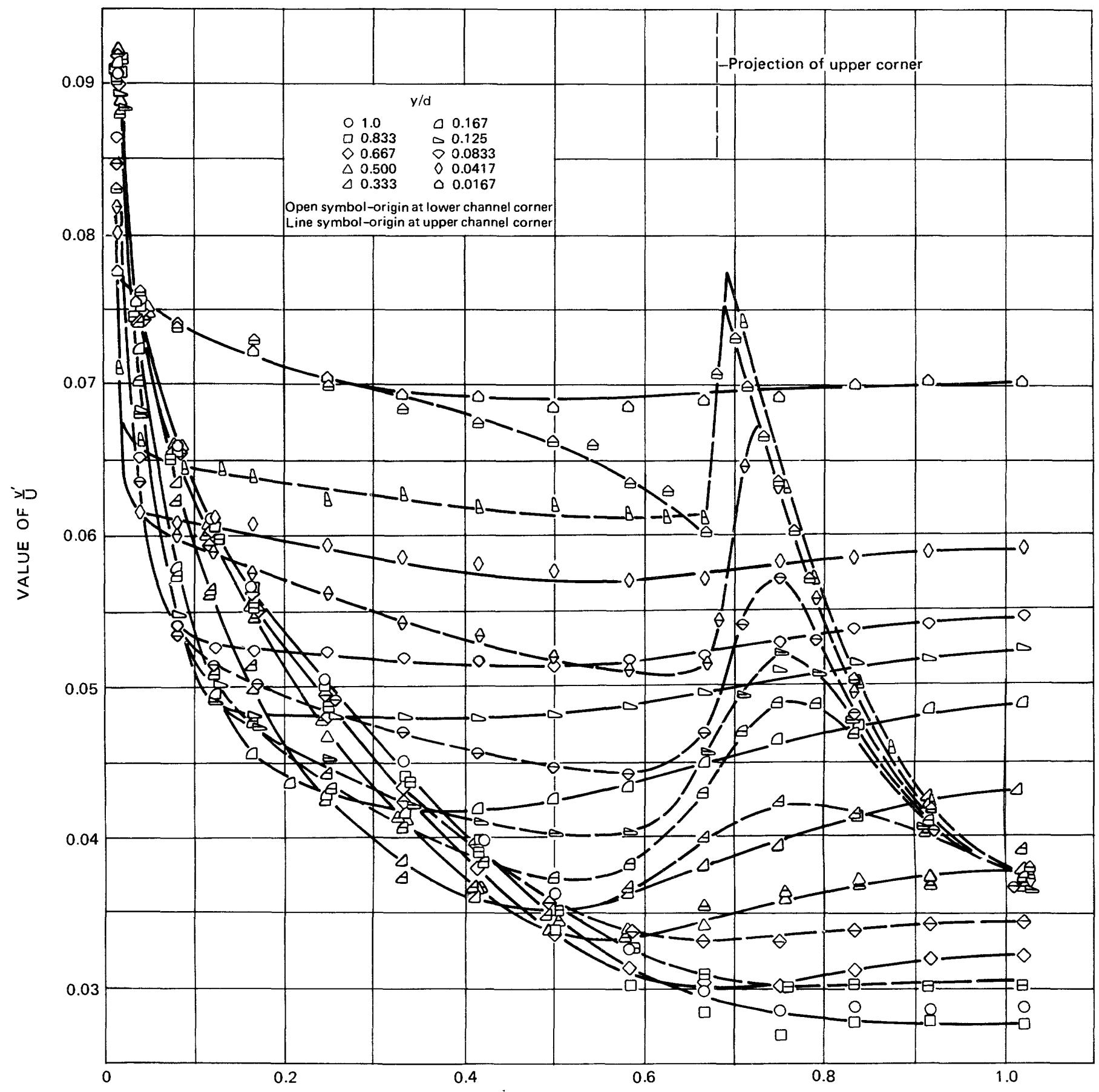

VALUE OF $z / d$

FIGURE 8.-Distribution of $v^{\prime} / U$.

at the upper and lower corners, as for figures 5-8. The signs of the stresses, where they depend upon the vertical coordinate, are assigned on the basis of the lower origin, with $y$ increasing continuously from the lower to the upper boundary.

The quantities $\rho \overline{u v}, \rho \overline{u w}$, and $\rho \bar{v} w$ are physically sig- nificant as tangential, or shearing, stresses, which result from the turbulent interchanges of mass and momentum between neighboring flow regions. The concept, and its details, are well documented in hydraulic literature. The exchanges, in all directions, lead to the stress system shown in figure 1 . The sign of 


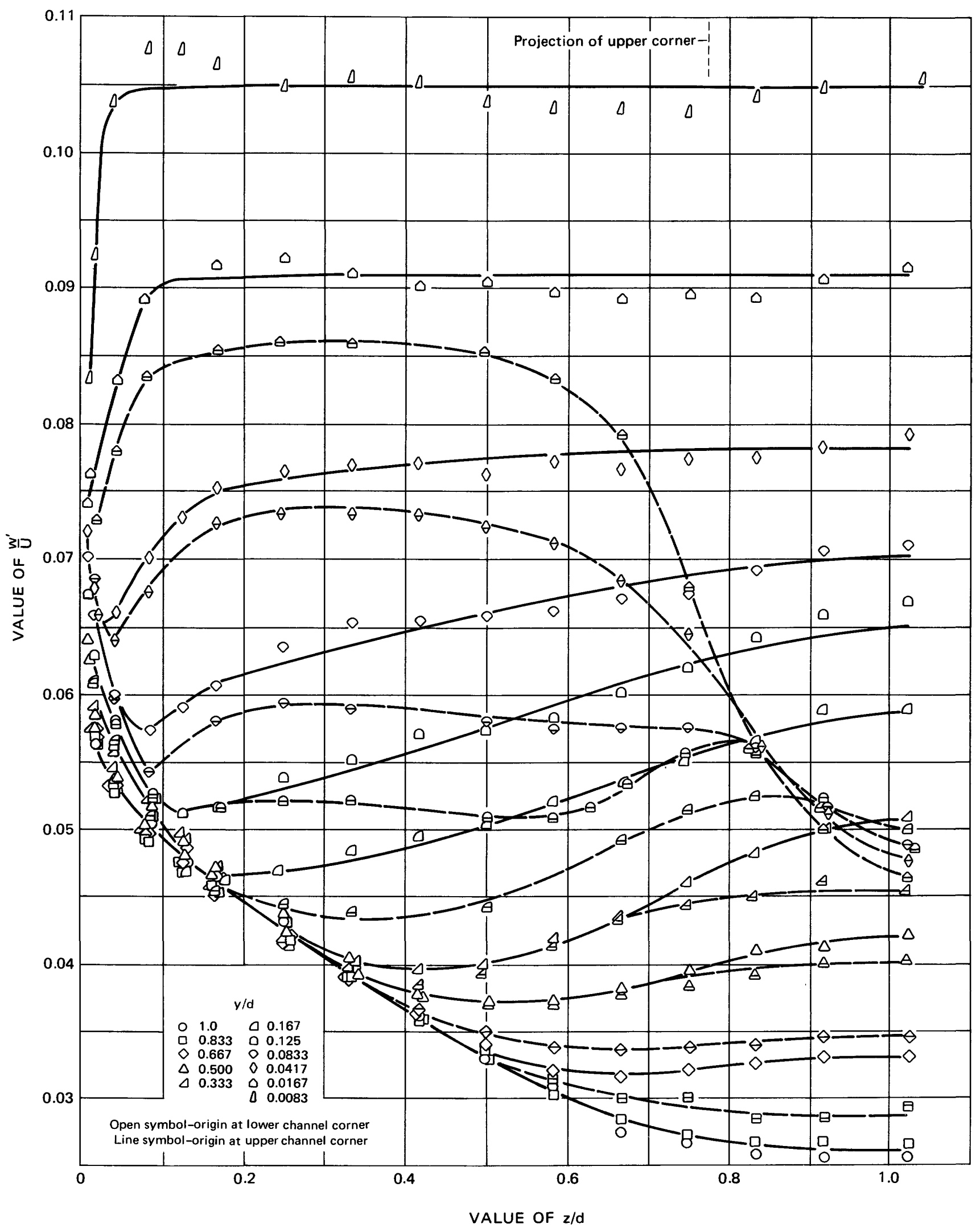

FIGURE 9.-Distribution of $w^{\prime} / U$. 
RESULTS

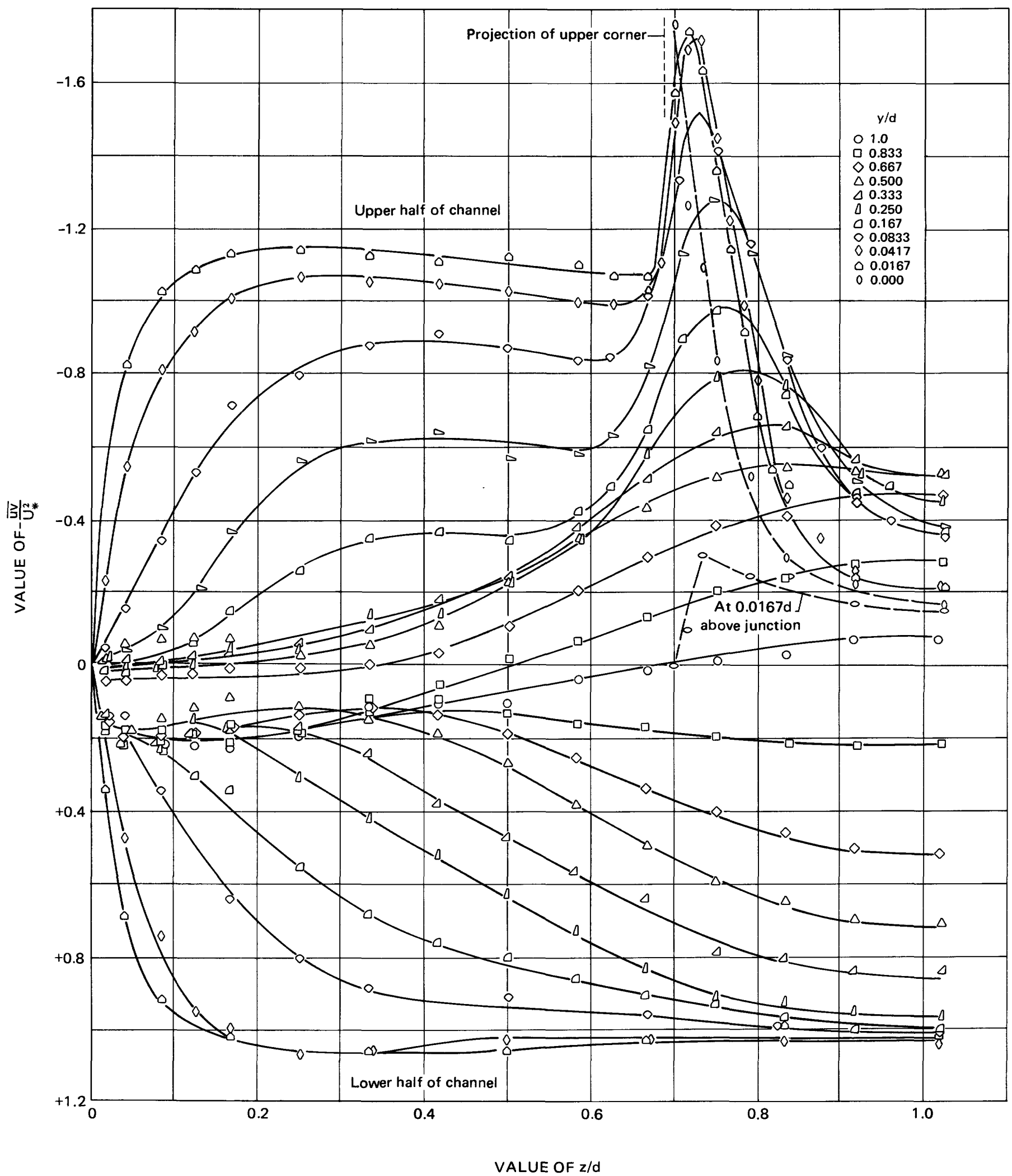

Figure 10.-Distribution of $\overline{u v} / U_{*}{ }^{2}$. 


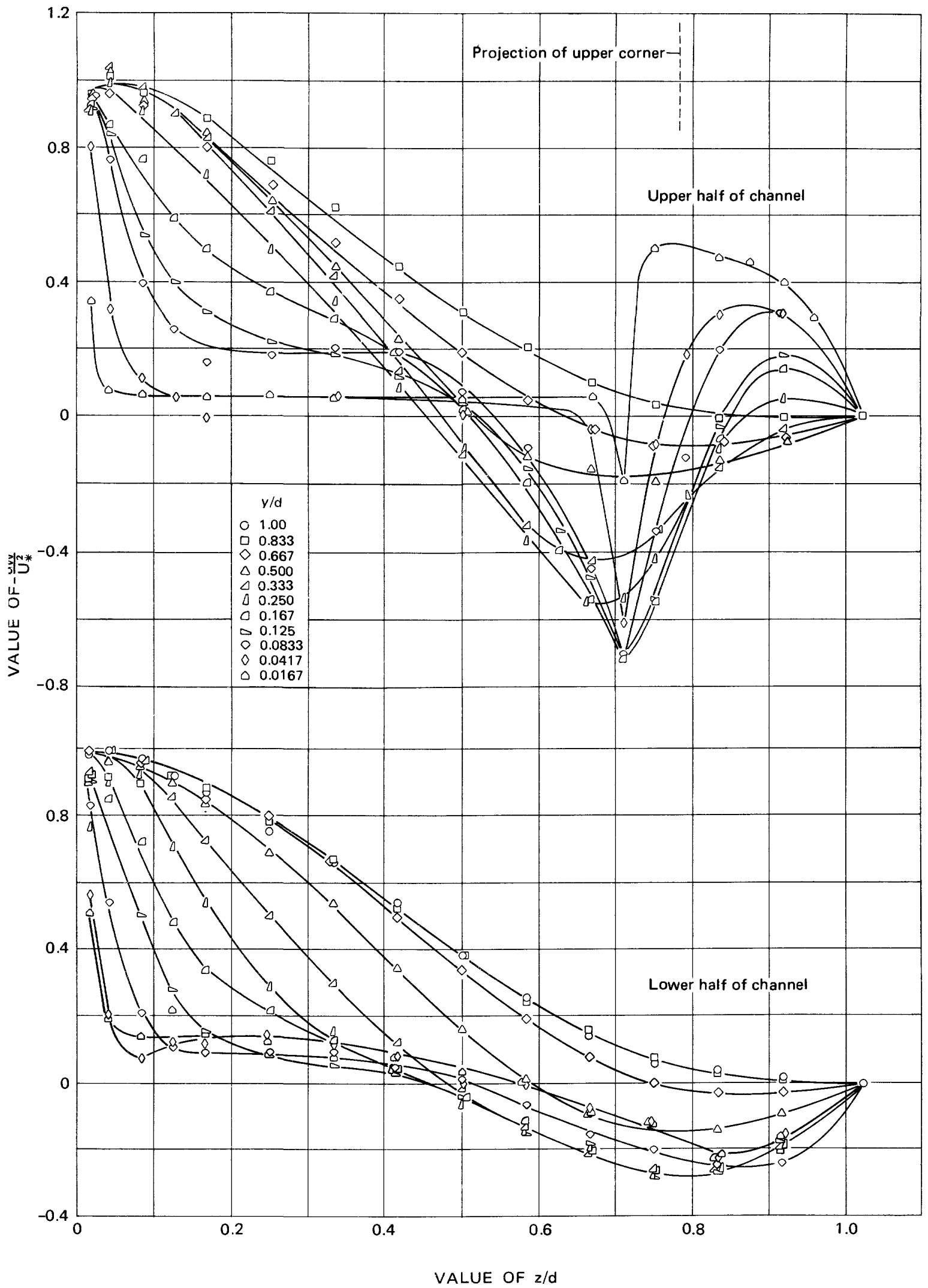

FIgURE 11.-Distribution of $\overline{u w} / U_{*}{ }^{2}$. 


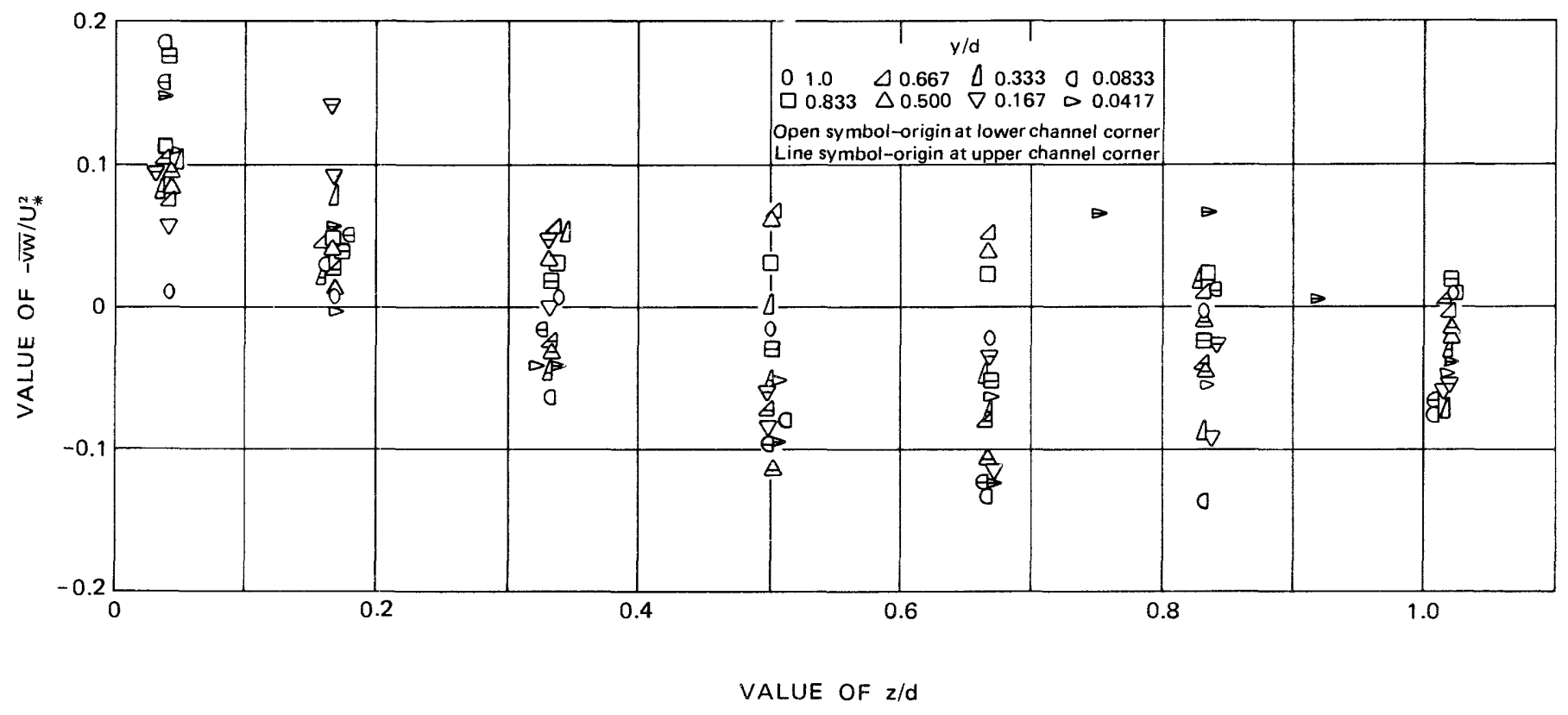

Figure 12.-Distribution of $\overline{v w} / U_{*}^{2}$.

the stress is a function of the mean velocity gradient in the direction of the transfer. The nature of the transfer process is to produce a negative correlation between the two members of the shearing-stress terms for a positive gradient-that is, a velocity increasing in a positive direction outward from a boundary.

The signs of the tangential stresses are related, also, to the rotational aspects of the stresses. Thus, each of the tangential stresses-and as well, the normal stresses-is opposed by its counterpart on the opposite, parallel face of figure 1 . The tangential components in opposite directions along parallel faces are stress couples which tend to rotate the particle. The sign of the stress component indicates the angular direction of the rotational tendency, which can be shown to be a function of the velocity gradient normal to the stress couple. Because the stresses are negative for a positive gradient, they are shown with negative signs on figures 10,11 , and 12 .

The surface force due to a stress is the product of the stress intensity and the area of the face over which it acts. The resultant surface force on the particle of figure 1 due to a given stress component is the difference between the oppositely directed forces on the opposite, parallel faces, and is conveniently expressed in gradient form. The resultant surface force, per unit of volume, of a tangential stress is its gradient normal to the stress couple. For a normal stress, the resultant, also per unit of volume, is the gradient of the stress in the direction of the stress. The resultant of all surface forces due to the turbulent exchanges, per unit of vol- ume, in the $x, y$, and $z$ directions are the turbulence terms on the right side of equations (2), (3), and (4). For the case of a uniform motion, they are given by the right side of equations (6), (7), and (8).

The total intensity of shear is the sum of the turbulent stress and that due to direct viscous action. The second is small relative to the first, except at locations where the mean velocity gradients are large. In the test channel, the gradients are large near the boundaries. Elsewhere, the turbulent stresses are a close approximation to the total stress. By ignoring the decreasing portion of the turbulent stress distributions near the boundaries, the distributions of figures 10 and 11 may be projected to the boundaries to obtain an estimate of the value of the total stress at the boundary. The value of $\overline{u w} / U *^{2}$ projected in this manner to the vertical boundary of the larger section is remarkably constant over almost the entire length of the boundary (the immediate corner regions are the exception) and equal to 1.13. A corresponding projection of $\overline{u v} / U *^{2}$ to the upper and lower boundaries is similarly constant and equal to 1.17 and 1.11 , respectively. Measurements made in the upper channel (not shown) yield boundary values smaller than these, equal to 0.61 on the horizontal boundary and 0.76 on the vertical wall. An average, obtained by weighing the boundary stresses for the large and small channel against peripheral length, is 1.01 , which is a favorable comparison between the average boundary stress computed from the hot-wire measurements and from the piezometric grade line. The comparison serves as a 
general check on the hot-wire techniques used during the study and on the magnitude of the stress terms. It is suggested, moreover, that the apparent constancy of the boundary shear stress over large segments of the test channel periphery may be typical of flow in more complex channels. This observation is substantiated by the successful use of equations of the Manning or Chezy type in the large variety of channels to which they have been applied.

Because of the dependency of the sign of the stresses upon a mean velocity gradient and because of the analogy with laminar flow, in which the stress is directly proportional to the gradient, attempts have been made to relate the magnitude of the turbulent stresses to the magnitude of the velocity gradients, without complete success. An example is shown in figure 13, on which is plotted the variation of $\overline{u v} / U_{*}^{2}, \partial U / \partial y$, and $\overline{u v} / u^{\prime} v^{\prime}$ for $y / d=0.167$ in the upper half of the channel. The variables are imperfectly correlated, and over a part of the length of the curves, $\partial U / \partial y$ actually is decreasing while $\overline{u v}$ is increasing.

In explanation, Schubauer and Klebanoff (1951), point out that a fundamental difference between laminar and turbulent flow is the scale of the mixing, that for turbulent flow being much larger. Their measurements of correlation distance show that the large-scale components of the eddies occupy a large portion of the width of the channel. Their simplified model describes a transfer of energy from larger to successively smaller eddies, the action of viscosity becoming progressively greater with diminishing size. Because the largest eddies thus make the greatest contribution to the average shearing stress, they suggest that the stress should be expected to depend upon a velocity gradient averaged over a distance, rather than upon the local gradient at a point.

While the foregoing is reasonable, the selection of an averaging distance to reconcile the velocity gradients with observed values of the turbulent stress is not obvious. According to figure 13, the distance is evidently variable, depending upon location. It is also frequently zero. In figure 11 and figure 6 , for example, $\overline{u w}$ and $\frac{\partial U}{\partial z}$ are perfectly correlated in the numerous instances where both are equal to zero (in addition to the obvious case at $z / d=1.0$ ) at identical locations. It thus appears that a simple method of predicting the magnitude of the turbulent stress from the mean velocity variation is not at hand.

The variation of $\overline{u w}$ and $\overline{u v}$ over the section creates force components that frequently act in the same direction as the pressure force-that is, a "negative" shearing force. Reversals in the direction of the forces occur at changes in the sign of the gradients $\frac{\partial \overline{u v}}{\partial y}$ and $\frac{\partial \overline{u w}}{\partial z}$
Figure 11 shows many such locations for $\frac{\partial \bar{u} \bar{w}}{\partial z}$ for $0.7<z / d<0.9$. The reversals in the sign of $\frac{\partial \overline{u v}}{\partial y}$ (fig. 10) are less evident, because the variation of $\overline{u v}$ with $y$ is not shown. The gradient does change sign, however, at many locations in the vicinity of the 260-degree corner.

An inspection of figures 10 and 11 also shows that the stress gradients become large in all corner regions. The combination $\left(-\frac{\partial \overline{u v}}{\partial y}-\frac{\partial \overline{u w}}{\partial z}\right)$ of stress gradients produces large positive forces (in opposition to the pressure force) in the 90-degree corner regions and large negative forces in the 270-degree corner regions, and at locations near the vertical axis of symmetry of the channel. A numerical evaluation of the forces in the upper half of the channel is given in the last section of the report.

The shear-stress variation at the junction of the larger and the smaller channel is pertinent to the channel subdivision procedures commonly used in engineering practice. The procedure does not consider the junction to be a shear surface and hence omits its length from the "wetted" perimeter of each subsection, which is to say that the shear-stress component normal to the junction $(\rho \overline{u v})$ is assumed to be zero everywhere along the junction.

A reference to figure 10 indicates that $\rho \overline{u v}$ is not zero at the junction; in fact, it is large over the portion adjacent to the apex of the corner. Figure 10 also indicates that the stress decreases rapidly with distance above the junction, however, and at $y / d=0.0167$ above the junction it is almost insignificant. The large stress values at the corner are probably due to locally large values of $\frac{\partial U}{\partial y}$ at the corner. If the locally large values of the stress are discounted, the zero stress assumption is probably more valid than one in which the junction is treated as a solid boundary.

The values of $\overline{v w} / U_{*} 2$ of figure 12 depend upon the separation of a small difference between two large signals, each of which is characterized by some degree of uncertainty. The individual measurements must therefore be accepted with reserve. The measurements, together, probably can serve to indicate the order of magnitude of the stress with more confidence, and the figure is shown primarily for this purpose. No average lines have been drawn through the points.

The $\overline{v w}$ component of stress is a momentum exchange which depends upon the intensity of its turbulent components and upon some function of the gradients $\partial V / \partial z$ and $\partial W / \partial y$. The gradients may be appreciable, even though $V$ and $W$ are small relative to $U$.

The correlation coefficients $\overline{u v} / u^{\prime} v^{\prime}$ and $\overline{u w} / u^{\prime} w^{\prime}$ are shown in figures 14 and 15. Maximum values of the 


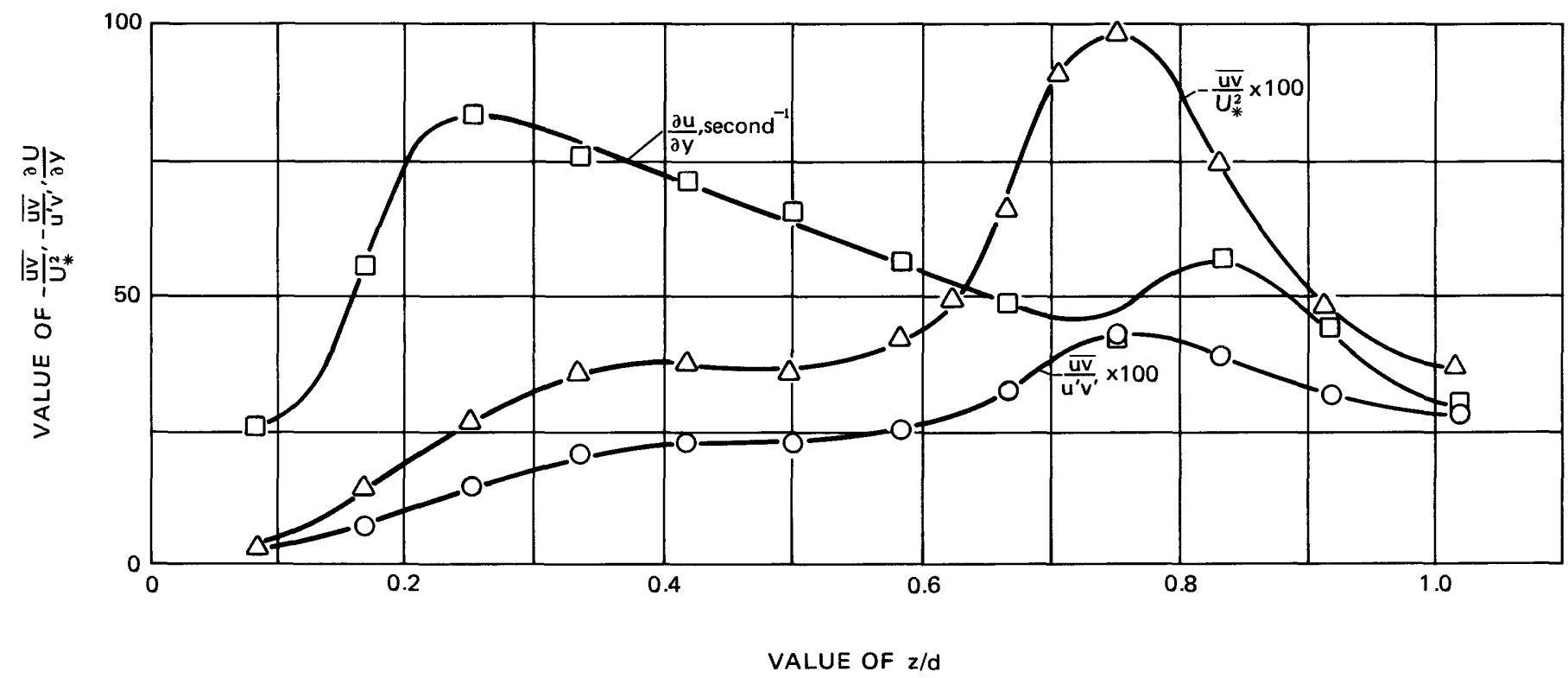

FIGURE 13.-Comparison of velocity gradient, turbulent shear-stress, and correlation coefficient at $y / d=0.167$ in upper half of channel.

coefficients are dependent upon Reynolds number (Laufer, 1951). The maximum values in figures 14 and 15 (about 0.4 ) are close to those obtained by Laufer at a similar Reynolds number in a one-dimensional flow, except at the 270-degree corner, where they are greater in the case of $\overline{u v} / u^{\prime} v^{\prime}$.

\section{SECONDARY MOTION PATTERN}

The secondary motions in the section of the nearly square lower channel are shown in figure 16 . The vectors of the figure are the combination of separate evaluations of $V$ and $W$. The untipped end of each vector represents the point at which the measurements were made, and the length of the vector is proportional to the velocity. A reference vector equal to $0.0133 U_{o}$ ( $1 \mathrm{ft} / \mathrm{s}, 0.305 \mathrm{~m} / \mathrm{s}$ ) is shown on the figure.

The secondary velocity components are difficult to measure accurately because of their small magnitude. On the other hand, the measurements are relatively easy to obtain, and so each was repeated several times to minimize error sources, such as voltage drift.

The void space in the center of the figure is an acknowledgment of a lower limit to the velocity signal separable from instrument noise and normal voltage drift. Although they probably are not zero, the motions in the region are small enough to be inconsequential. Elsewhere, the components were compared on the basis of continuity, using the relationship $\partial V / \partial y=-\partial W / \partial z$. From this standpoint, the measured motion pattern was reasonable everywhere except at the upper 90-degree corner, where the motions are small, and probably wall influenced, resulting in poor definition in this area.
The secondary motions consist of one large cell in the upper and in the lower part of the channel and a smaller cell alongside each of the larger eddies. The adjacent cells are counterrotating. The magnitude of the motions in the lower half of the channel are comparable to those of an earlier study (Tracy, 1965) in a narrow, rectangular channel at a Reynolds number of 96,000 and to those in a square channel at a Reynolds number of 83,000 (Brundrett and Baines, 1964). The Reynolds number of the present study is about 450,000 .

From this data, and the fact that the motions in the upper large cell near the 270-degree corner are larger than those in the lower cell by a factor of almost three, it appears that the boundary configuration in the vicinity of the motion is a more decisive influence upon the strength of the motion than is Reynolds number. It may be conjectured, also, that the effects of a given boundary feature are not entirely local. The differences between the secondary motions in the upper and lower 90-degree corner regions are probably an indication that the strong motions in the vicinity of the 270 degree corner affect those of the upper 90-degree corner.

In any event, the measured pattern is at variance with the conventional view held of the motions. They are usually described in terms of symmetrical rotations in cells bounded by the corner bisectors, the rotation having the opposite sense in adjacent cells. The measured motions are symmetrical only in a local region about the bisector of the lower corner, and because of the poor definition at the corresponding upper corner, they may also be locally symmetrical there. The motions at the 270-degree corner are divided into 


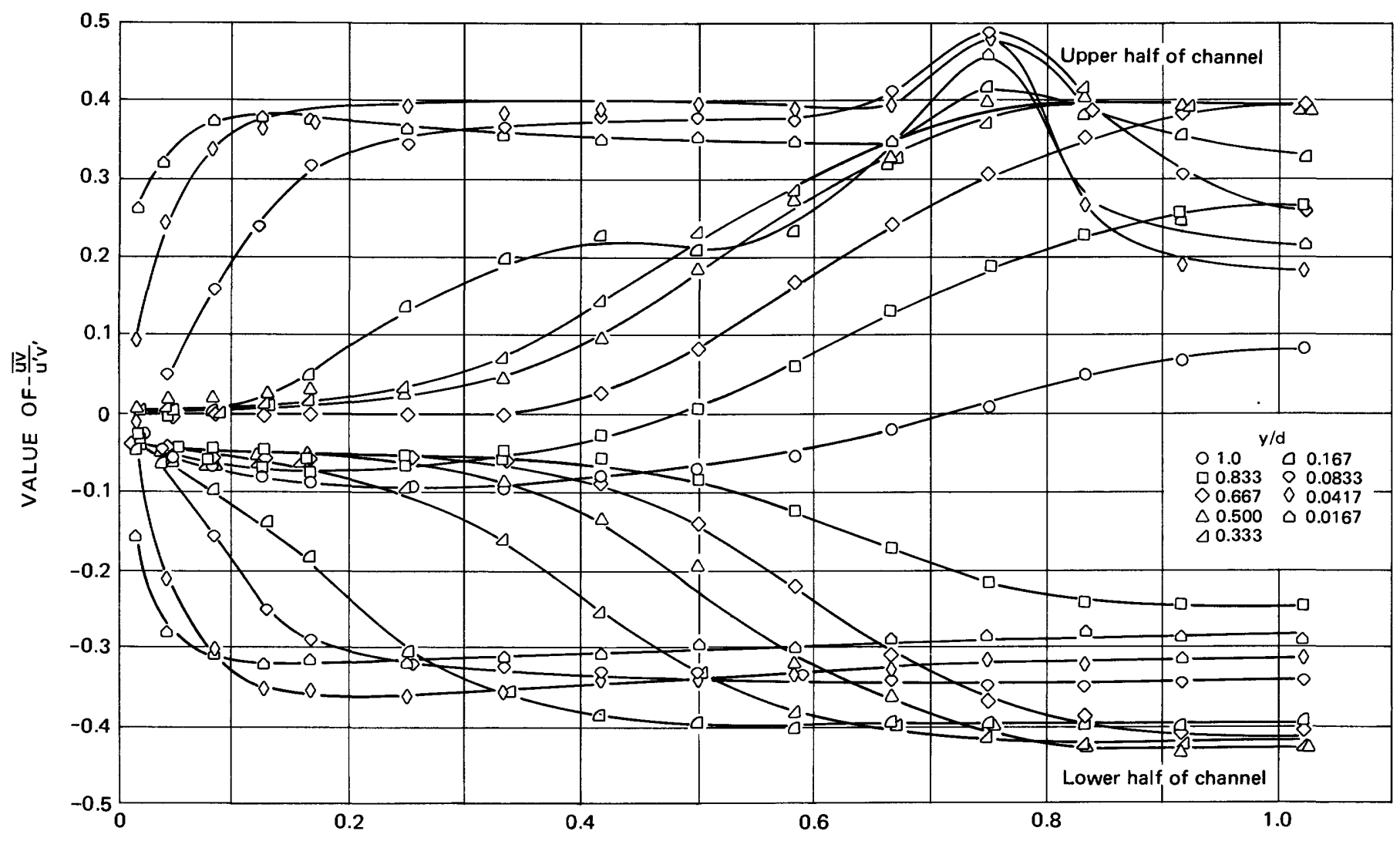

VALUE OF $z / d$

FIGURE 14.-Correlation coefficient $\overline{u v} / u^{\prime} v^{\prime}$.

two distinct circulations; interestingly, they are directed toward the corner along the boundaries and outward elsewhere, in contrast to the motions at the interior corners. The bisector, however, only approximately divides the cells.

The peculiarities in the distributions of longitudinal velocity of figure 6 are consistent with the measured secondary motion pattern.

The exchange of fluid between the two parts of the channel of their junction is not zero. Fluid is transported out of the larger channel at the center, and an equal amount is returned near the boundary.

\section{ORIGIN OF THE SECONDARY MOTIONS}

The secondary motions are believed to have their origin in the characteristic behavior of the normal stress components $\bar{v}^{2}$ and $\overline{w^{2}}$ in the corner regions of noncircular conduits. The typical unbalance of the components near a boundary has been outlined in an earlier section of the report.

A connection between the normal stresses and the secondary motions can be established by a minipulation and combination of equations (7) and (8), which describe the forces and the resultant accelerations in the plane of the secondary motions. The pressure terms may be eliminated by the differentiation of each equation with respect to the direction of action of the other and by the subsequent subtraction of one from the other. A further use of equation (5) produces the following result:

$$
\begin{aligned}
\rho V \frac{\partial \xi}{\partial y}+\rho W \frac{\partial \xi}{\partial z}=\frac{\partial^{2}}{\partial z^{2}} \rho \overline{v u}-\frac{\partial^{2}}{\partial y^{2}} \rho \overline{v w} & +\frac{\partial^{2}}{\partial y \partial z} \rho\left(\overline{v^{2}}-\overline{w^{2}}\right) \\
& +\mu\left(\frac{\partial^{2} \xi}{\partial y^{2}}+\frac{\partial^{2} \xi}{\partial z^{2}}\right)
\end{aligned}
$$

where $\xi=\frac{\partial W}{\partial y}-\frac{\partial V}{\partial z} \mid$ and is a measure of the rotation of a fluid particle about an axis normal to the $y-z$ plane.

Equation (9) may be obtained in an alternative fashion. Brundrett and Baines (1964) suggest that it can be derived directly by equating the rate of change of angular momentum of a volume of fluid-the terms on the left side-to the moments of the surface forces on the volume, which are the terms on the right side.

A laminar, uniform state of motion is described by 


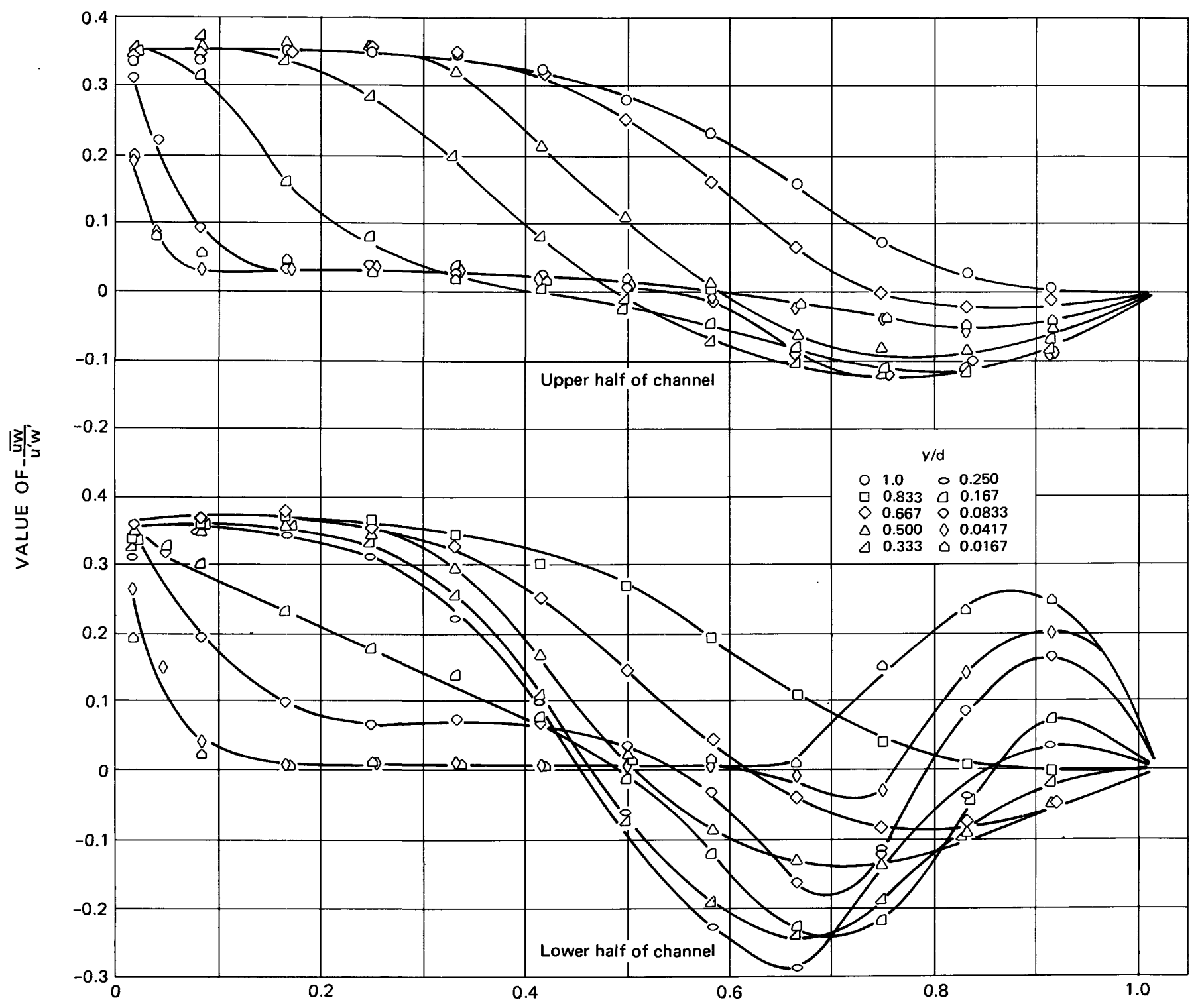

VALUE OF $z / d$

FigURE 15.-Correlation coefficient $\overline{u w} / u^{\prime} w^{\prime}$.

the accelerative terms on the left of equation (9) and by the last term on the right. Using the laminar condition as a starting point, Maslen (1958) demonstrated mathematically that if the secondary components are zero anywhere in a viscous motion, they are zero everywhere. Because they are zero at a boundary, the transverse velocities must vanish in a uniform, laminar flow. The terms involving the viscosity are thus counterproductive to the generation of the secondary motions.

The relative importance of the terms containing $\overline{v w}$ - the first and second terms on the right of equation (9)-are difficult to assess experimentally because of the small magnitude of $\overline{v w}$ and the probable measurement error. For the purpose of eliminating $\overline{v w}$ as a possible cause of the secondary motions, it probably suffices to remark that the stress depends upon the existence of $V$ and $W$ (or, more strictly, upon their gradients) and not the reverse. It is to be noted, moreover, that the $\overline{v w}$ terms of equation (9) are the turbulent counterparts of the viscous terms and may reasonably be expected to behave in a parallel manner-that is, to retard the rotation of the fluid particles.

To the extent that the latter conclusion is justified, the remaining term on the right side of equation (9), which is descriptive of the action due to the forces $\overline{v^{2}}$ 


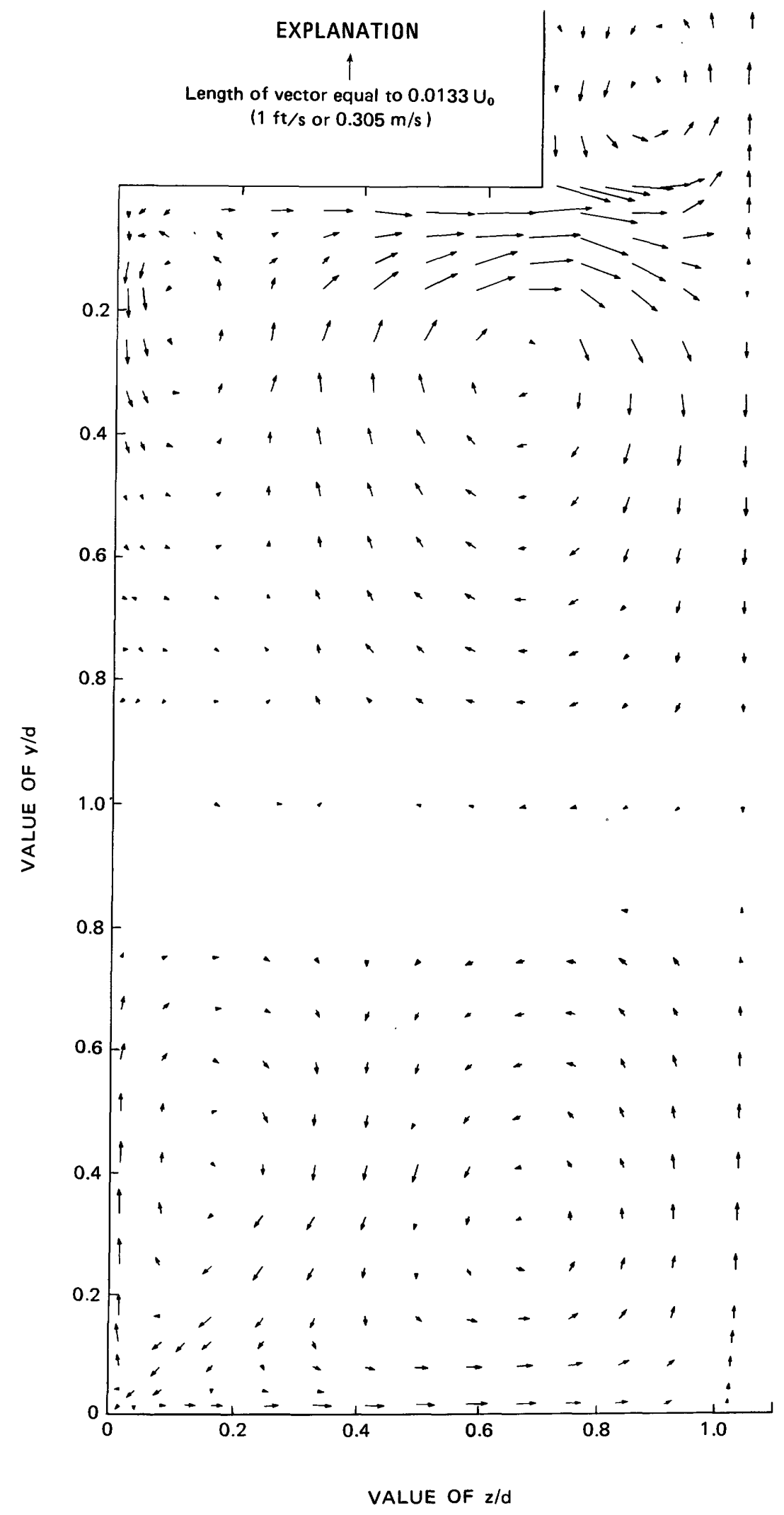

FIGURE 16.-Secondary motions in channel. 
and $\overline{w^{2}}$, is evidently responsible for the existence of nonzero values of the accelerative terms on the left and thus for nonzero values of $V$ and $W$. The magnitude of $\bar{v}^{2}$ and $\bar{w}^{2}$ is a function of location, Reynolds number, and boundary configuration and roughness. It is remarked that the structure of $\frac{\partial^{2}}{\partial y \partial z}\left(v^{2}-\overline{w^{2}}\right)$ (or of its equivalent in cylindrical coordinates) precludes a nonzero value of the term in an axially symmetric or twodimensional flow. The observation that secondary motions are not present in these types of flow is support for the conclusion just drawn.

The variation of $\overline{v^{2}}-\overline{w^{2}}$ over the section of the large channel is shown in figure 17. The measurements of $\overline{v^{2}}$ at $y / d=0.0016$ in both the upper and the lower half of the channel appear erroneous, and the probable value of $\overline{v^{2}}-\overline{w^{2}}$ at these locations is indicated as a dashed line. The principle function of figure 17 is to show that the velocity fluctuations in the plane of the channel section $-v$ and $w$-vary in a consistent manner near a boundary, with the component of turbulence normal to the wall always being smaller than the component which is parallel to the wall, and that the typical variation will always produce a nonzero value of $\left.\frac{\partial^{2}}{\partial y \partial z} \overline{\left(v^{2}\right.}-\overline{w^{2}}\right)$ in a corner region.

The result of the combination of boundary effects on the variation of $\frac{\partial^{2}}{\partial y \partial z}\left(\overline{v^{2}}-\overline{w^{2}}\right)$ is shown in figure 18 for the upper one-half of the larger channel. The correlation of the magnitude of the derivative with the magnitude of the secondary motions is good (see fig. 16).

\section{ROLE OF THE SECONDARY MOTIONS}

The turbulent shear gradients, and thus the turbulent shear forces, become very large in the corner regions of the channel. Without the secondary motions, which compensate for the force excess, the motion pattern in the channel would be greatly different from that which actually exists. The role of the motions is thus an essential one. It is described by equation (6) which expresses the longitudinal force balance in the channel:

$$
\begin{array}{r}
\rho\left(V \frac{\partial U}{\partial y}+W \frac{\partial U}{\partial z}\right)=-\frac{\partial p}{\partial x}+\mu\left(\frac{\partial^{2} U}{\partial y^{2}}+\frac{\partial^{2} U}{\partial z^{2}}\right) \\
-\rho\left(\frac{\partial \overline{u v}}{\partial y}+\frac{\partial \overline{u w}}{\partial z}\right) .
\end{array}
$$

The terms on the left, excluding $\rho$, are the longitudinal acceleration of a volume of fluid. The terms on the right, in order, are the forces due to pressure, viscosity, and turbulent shear, all per unit of volume. The negative pressure gradient indicates a decreasing pressure in the downstream direction. The negative turbulent shear terms are in recognition of the forementioned fact that the turbulence components normally correlate in the negative sense.

Despite their accelerative significance, and like the turbulent products, the terms on the left side of equation (6) can also be interpreted as forces due to momentum transport. Consider, for example, the term $\rho V \frac{\partial U}{\partial y}$, in which $V$ is a volume rate of vertical flow per unit of area. As the volume is carried from one region to another-the $U$ velocity generally being different in the two regions-it undergoes a momentum change per unit of volume equal to $\rho \frac{\partial U}{\partial y} d y$ in the distance $d y$. In contrast to a turbulent fluctuation, which is assumed to retain its $U$ velocity throughout its migration, the volume transported by $V$ undergoes a continuous change in $U$. The integral of the product of $V$ and $\rho \frac{\partial U}{\partial y} d y$ is thus the rate of transport of momentum per unit of area as a result of the secondary motion $V$ and is equivalent to the stress intensity due to the momentum exchange. The resultant surface force per unit of volume is the differential, with respect to $y$, of the integral, which is $\rho V \frac{\partial U}{\partial y}$ and which is the first term of equation (6).

The viscous forces are small relative to the other forces except near a boundary where the velocity derivatives upon which they depend are large. At a boundary distance equal to $0.07 d$, the viscous forces are typically less than 3 percent of the pressure force. The viscous forces have been omitted from consideration at greater distances.

Thus, not too close to the boundaries, equation (6) is a balance between the momentum transfer due to turbulence, the transfer due to the secondary motions, and the pressure force. Because the pressure force is everywhere constant over the section, a variation in one of the transfer forces must be accompanied by an opposite variation in the other.

The forces have been evaluated from experimental data at $y / d=0.0833$ in the upper and in the lower parts of the section and are shown in figure 19. To facilitate comparison, the negative of the secondary motion trasnport forces have been plotted in the figure. (To be noted, also, is the division of each term of equation (6) by the mass density, $\rho$, so that the force significance of each is lost. The result, however, is proportional to force.) 


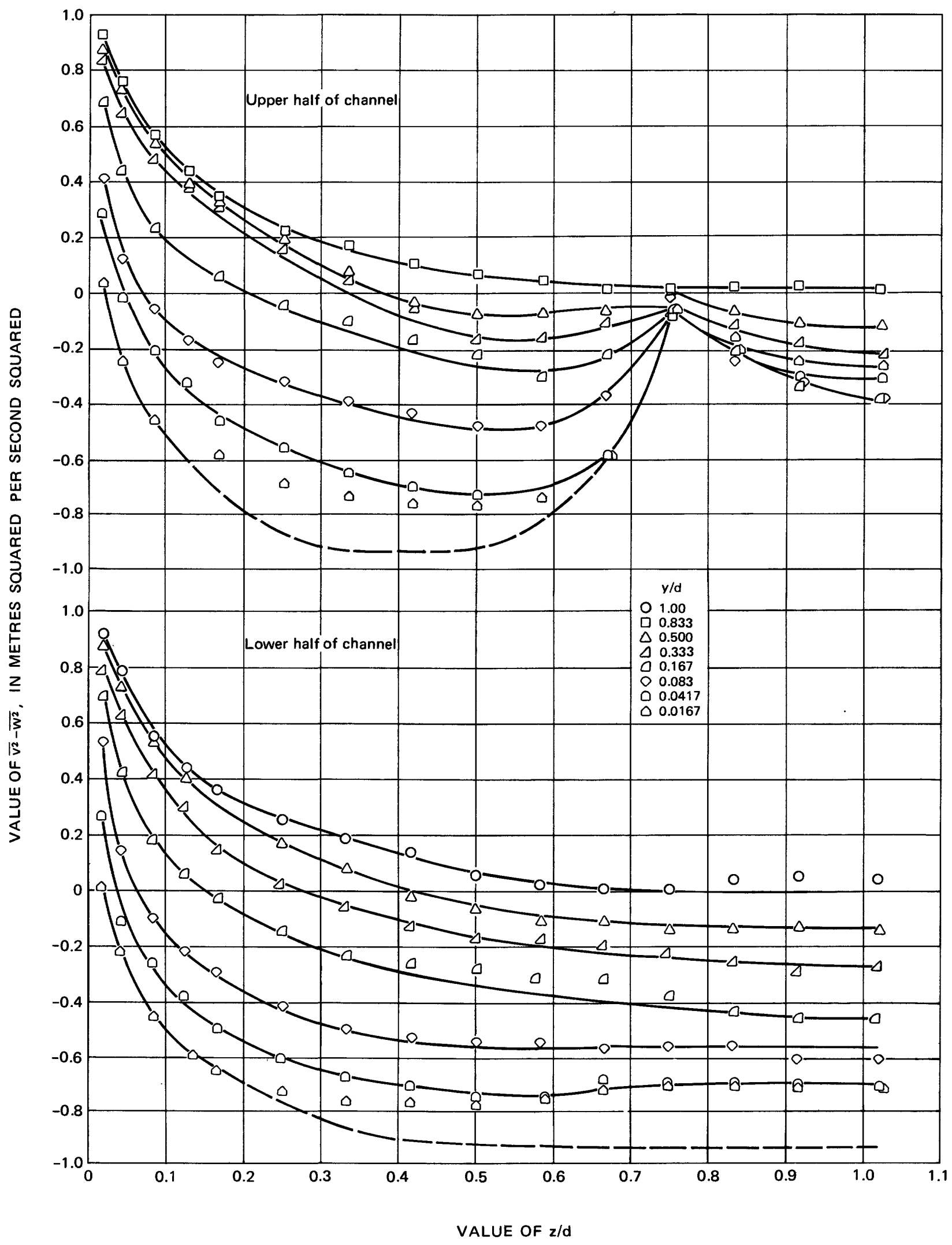

Figure 17.-Distribution of $\overline{v^{2}}-\overline{w^{2}}$. 
ROLE OF THE SECONDARY MOTIONS

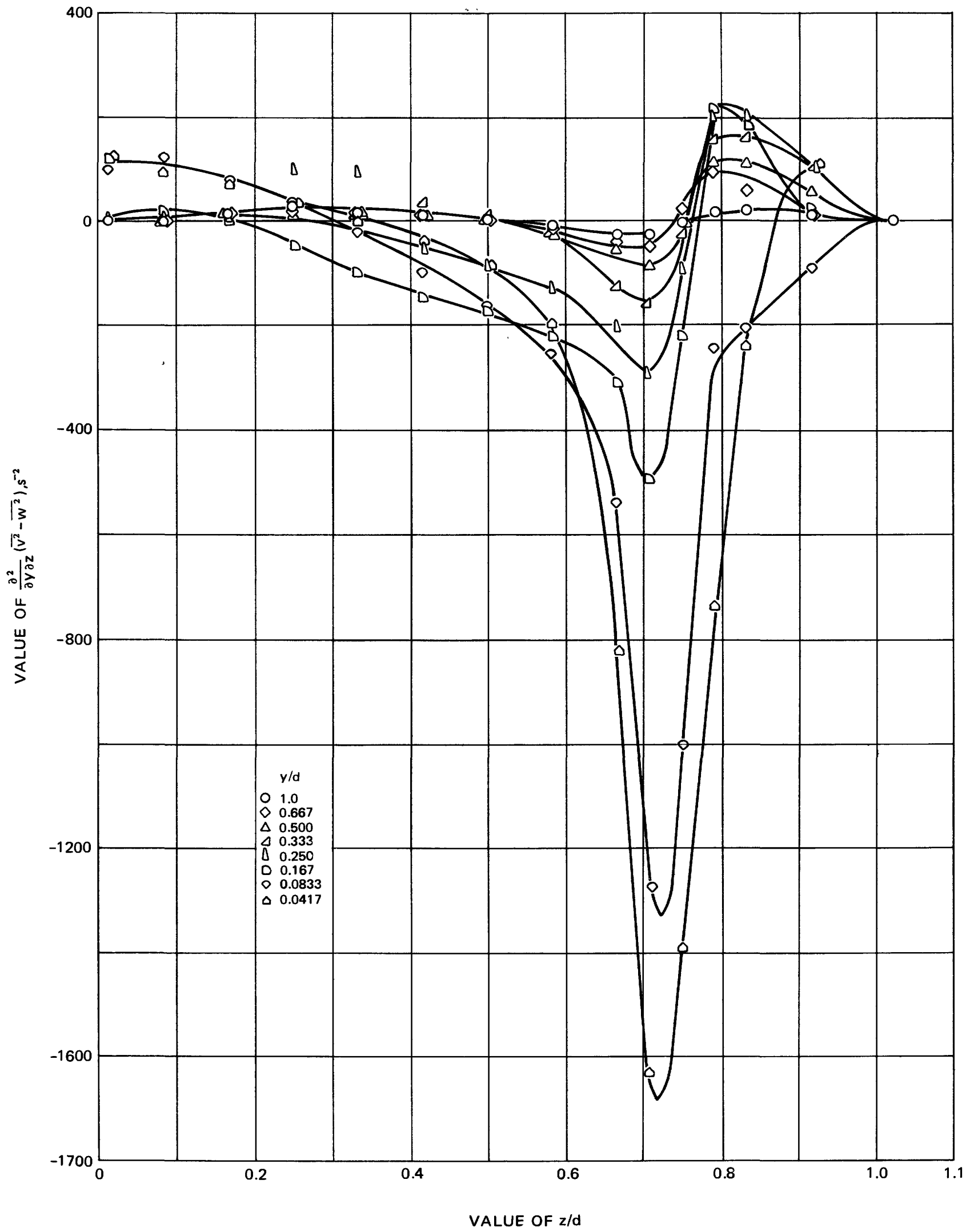

FIGURE 18.-Variation of $\frac{\partial^{2}}{\partial y \partial z}\left(\overline{v^{2}}-\overline{w^{2}}\right)$. 


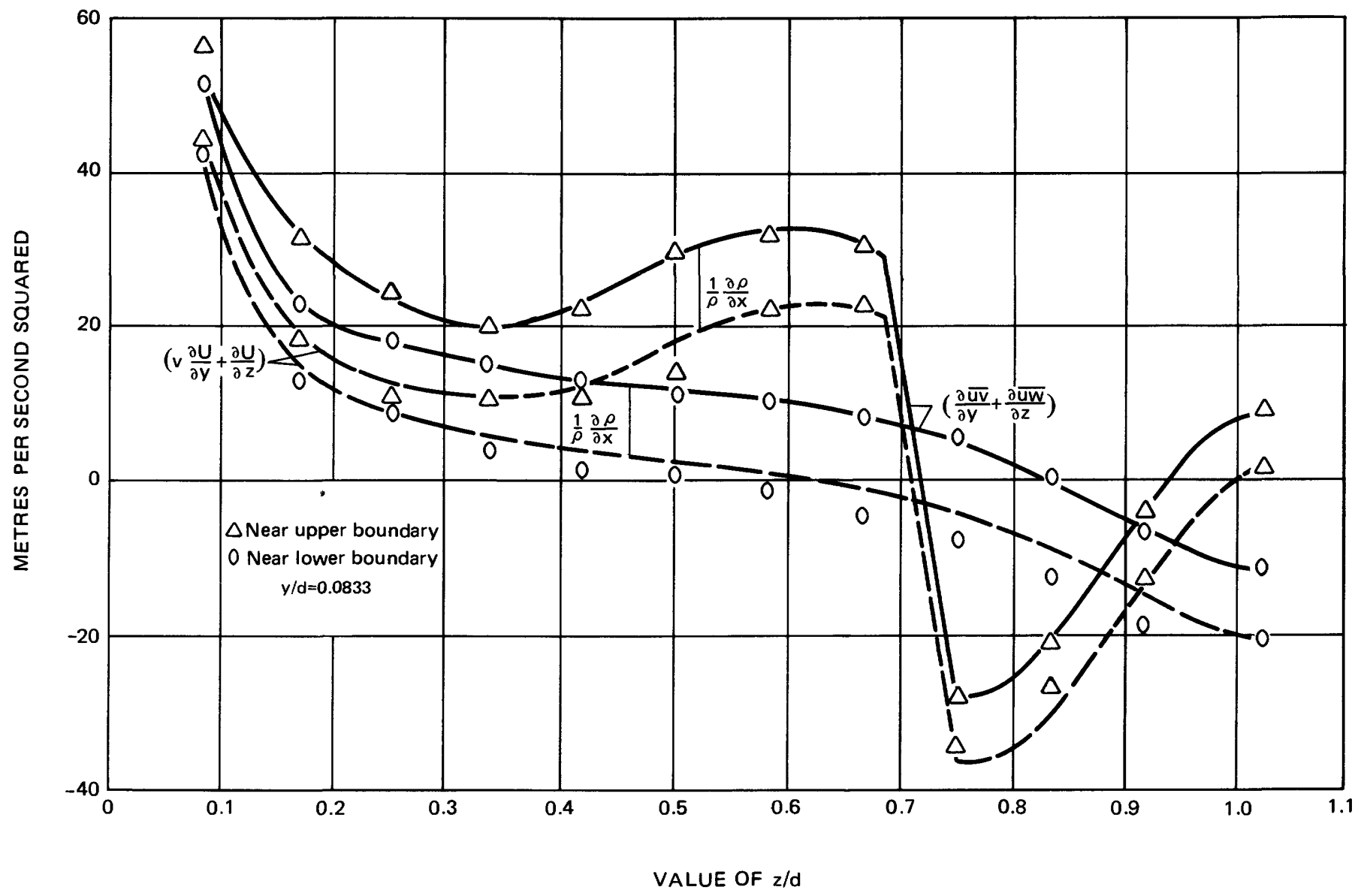

Figure 19.-Balance of first equation of motion at $y / d=0.0833$.

The force variation shown in figure 19 is a function of the details of the motion pattern in the vicinity of the variation. The motion pattern is described primarily by boundary geometry, with Reynolds number of secondary importance. The extremes of variation of figure 19 occur near points of change in boundary alignment, or corners. To the extent that the corners are isolated from the direct influence of other corners, the motions and the resulting forces near each are "mostly" representative of the corner, although they are affected to some degree by every element of the boundary, however distant.

The 90-degree corners, for example, are characterized by large positive values of turbulent shear force, which are opposed by the pressure and the secondary motion transport forces. The large shear forces are the result of the typical variation of the turbulent shear stress components in such regions, illustrated by the lowermost of the curves of both parts of figure 11, which detail, for example, the variation of $\overline{u w}$ at small values of $y / d$. Near the corner, the magnitude of the component of shear stress at a point is a function of the relative proximity of the two walls to the point, the closer wall having the greater effect. The stress at a point close to one wall is not greatly affected by the opposite wall until the point is located equally as close to the latter. As the opposite wall becomes effective, the stress changes rapidly. To be noted in figure 11 are the large stress gradients near the vertical wall. Similar gradients of $\overline{u v}$ in the vertical direction account for the large shear force values in figure 19 . The secondary motions, and the forces therefrom, are the consequence of the difference in $\overline{v^{2}}$ and $\overline{w^{2}}$ at the boundaries and the reversal in the sign of the difference at the corner.

A "typical" force variation near the 270-degree corner is more difficult to describe, because of the influence of the other boundary elements on the more complex motion pattern. The effect of such an isolated corner may be approximated, however, as the difference between corresponding force distributions in the upper and in the lower part of the section at the same value of $y / d$. This assumes that the force distributions in the upper part of the section would be identical to those in the lower in the event that the junction between the two channel sections-the upper, smaller rectangular section and the lower, nearly square 
larger section-were formed of a solid, smooth material; a further assumption is that the distributions in the lower half of the larger channel would not be greatly affected by such a procedure. The first assumption is completely reasonable; the second is reasonable enough to indicate without serious error the trends and magnitudes of the forces for the replaced-boundary condition.

When compared in this manner, the trends shown by the distributions of figure 19 in the upper part of the section are largely unchanged. The effect of the corner is thus to cause a large change in the magnitude of both sets of the variable forces and to reverse the direction of each from one to the other side of the corner. At the left, the shear forces are large and are opposed by the pressure force. The momentum transport forces due to the secondary motions act in the direction of the pressure. Both forces are larger than the pressure force. At the right side, the roles are reversed: the shear forces now act in the same direction as the pressure, and the secondary motion force opposes each. Farther to the right, the forces again reverse.

At increased values of $y / d$, the corner influences lessen, and the forces decrease. At $y / d=1.0$, a twodimensional flow pattern is approximated, and the secondary motions are small or nonexistent. The two-dimensional counterpart of figure 19 is a straight horizontal line representing the value of $-\frac{\partial \overline{u w}}{\partial z}$ and is located a constant distance equal to $-\frac{1}{\rho} \frac{\partial p}{\partial x}$ above the line corresponding to a zero force.

The force variation for $y / d>0.0833$ in the upper half of the channel is illustrated in figure 20 . The sum of the turbulent exchange forces only is shown in the figure. The sum of $V \frac{\partial U}{\partial y}$ and $W \frac{\partial U}{\partial z}$ differ from corresponding values of these forces by the constant value of $-\frac{1}{\rho} \frac{\partial p}{\partial x}$, which is equal to $8.6 \mathrm{~m} / \mathrm{s} / \mathrm{s}$.

Figures 19 and 20 graphically depict the role of the secondary motions-that of an agency to equalize the large turbulent exchange forces in the corner regions. The turbulent forces can be much larger than the pressure force. If the motions did not exist, the fluid must stagnate and separate from the boundaries in the 90 degree corners and would be greatly accelerated in the vicinity of the 270-degree corner.

\section{CONCLUSIONS}

The experimental work reported herein is devoted to the internal details of a fully developed turbulent motion in a conduit of complex boundary form. The principal feature of the boundary configuration is a corner subtending an angle greater than 180 degrees. The results are principally concerned with the production and effect of the secondary motions in the conduit.

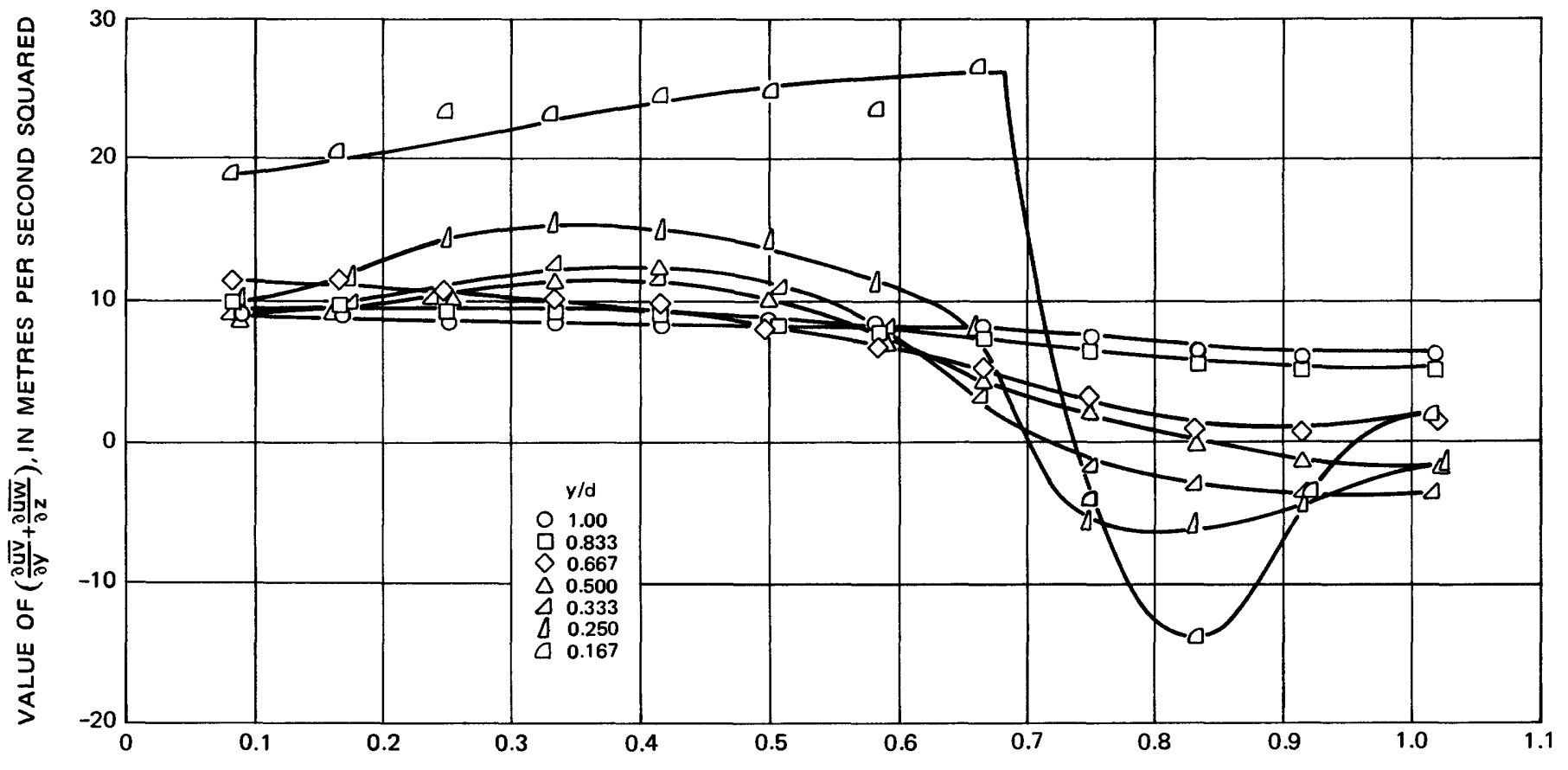

VALUE OF $z / d$

FIGURE 20.-Distribution of sum of turbulent shear forces. 
The following are the major results:

1. The variation of the turbulence components $v$ and $w$ in the corner regions of the conduit is the most probable cause of the secondary motions.

2. The magnitude of the secondary motions produced in a given corner region is a function of the angle subtended by the corner. The function may not be defined with the limited data at hand.

3. The secondary motions are an effective mechanism for the transfer of momentum in the plane of the channel cross section. The forces due to the momentum transfer are of the same order of magnitude as those due to turbulent shear and to pressure.

4. The combination of boundary effects at a corner produce turbulent shearing forces which are frequently larger than the force due to pressure. The shearing forces may oppose the pressure force or act in the direction of the pressure force, depending upon the corner configuration.

5. The secondary motions transfer an appropriately directed momentum into the corner regions to compensate for the excess of shearing force and, thus, to prevent flow separation or acceleration of the flow in the regions.

6. The secondary motions also, apparently, act to equalize the boundary shear stress over considerable portions of the boundary, accounting in large measure for the success of equations of the Manning or Chezy type applied to channels of complex form.
7. The transfer of fluid across the line separating the larger from the smaller channel is small. The shear stress at the line is also small, indicating that the channel subdivision practices commonly used are probably applicable, at least to the channel of this study.

\section{REFERENCES}

Brundrett, E., and Baines, W. D., 1964, The production and diffusion of vorticity in duct flow: Jour. Fluid Mechanics, v. 19, part 3, p. 375-392.

Hinze, J. O., 1954, Turbulence: New York, McGraw-Hill, p. 73-119.

Laufer, John, 1951, Investigation of turbulent flow in a twodimensional channel: Natl. Advisory Comm. Aeronautics report 1053, Washington, D.C.

1954, The structure of turbulence in fully developed pipe flow: Natl. Advisory Comm. Aeronautics report 1174, Washington, D.C.

Maslen, S. H., 1958, Transverse velocities in fully developed flows: Appl. Mathematics Quart., v. 16, p. 173-175.

Rodet, E., 1960, Etude de l'écoulement d'un fluide dans un tunnel prismatique de section trapézoidale: Ministére L'Air scientifiques et téchniques Pub. no. 369.

Schubauer, G. B., and Klebanoff, P. S., 1946, Theory and application of hot-wire instruments in the investigation of turbulent boundary layers: Natl. Advisory Comm. Aeronautics report W-86, Washington, D.C.

1951, Investigation of separation of the turbulent boundary layer: Natl. Advisory Comm. Aeronautics report 1030, Washington, D.C.

Tracy, H. J., 1965, Turbulent flow in a three-dimensional channel: Am. Soc. Civil Engineers Proc., Jour. Hydraulics Div., paper 4530 , v. 91, no. Hy 6, p. 9-35. 